University of Zurich

Department of Economics

Working Paper Series

ISSN 1664-7041 (print)

ISSN 1664-705X (online)

Working Paper No. 211

\title{
The Role of Trading Frictions in Financial Markets
}

Samuel Huber and Jaehong Kim

Revised version, July 2017 


\title{
The Role of Trading Frictions in Financial Markets*
}

\author{
Samuel Huber \\ University of Basel
}

\author{
Jaehong Kim \\ Xiamen University
}

July 21, 2017

\begin{abstract}
We develop a dynamic general equilibrium model to analyze the role of trading frictions in over-the-counter financial markets. In our model, agents face idiosyncratic preference shocks and a financial market allows them to rebalance their portfolio composed of liquid and illiquid assets in response to these shocks. We disentangle the effects of search and bargaining on welfare and study each one of them. We show that bargaining is welfare-improving for any inflation rate above the Friedman rule, while search frictions are not. This is because search frictions do not only affect the demand for the respective assets, but also their allocation.
\end{abstract}

Keywords: Monetary theory, over-the-counter markets, financial regulation, corporate bonds, liquidity.

JEL Classification: D47, D52, D62, E31, E44, E50, G11, G12, G28.

\section{Introduction}

Many financial assets are traded in over-the-counter markets, which feature search and bargaining. Due to the decentralized nature of these markets and the lack of publicly available trade information, they are sometimes also referred to as "dark markets" (see Duffie, 2012). To overcome these limitations, policymakers recently proposed measures to move some of these markets to a more centralized market structure. A case in point is the U.S. Securities and Exchange Commission, which, in 2014, promoted a more standardized issuance of corporate bonds in order to centralize corporate bond markets:

${ }^{*}$ We would like to thank Aleksander Berentsen, Christopher Waller, Pedro Gomis-Porqueras, Fernando Martin, and Cyril Monnet for very helpful comments. We also thank the Fundamental Research Funds for the Central Universities for financial support, Grant 20720171070. Samuel Huber is a research fellow at the Department of Economic Theory, University of Basel. E-mail: samuel_h@gmx.ch. Jaehong Kim is an associate professor at the Wang Yanan Institute for Studies in Economics and the School of Economics, Xiamen University. E-mail: jaehongkim@xmu.edu.cn. 
"I will not pretend that migrating fixed income transactions from the over-the-counter markets is a small task; however, I will note that in the early part of the twentieth century, there was an active market in corporate and municipal bonds on the New York Stock Exchange." (Gallagher, 2014)

In this paper, we take a closer look at trading frictions in financial markets and show under which conditions they are desirable. We derive these results in a dynamic general equilibrium model, where agents trade liquid for illiquid assets in response to idiosyncratic preference shocks. By liquid (illiquid), we mean that the asset can be used (cannot be used) to purchase consumption goods; i.e., that it serves as a medium of exchange. In our model, the liquid asset is fiat money and the illiquid asset is a one-period corporate bond, which is supplied endogenously by generic firms. The idiosyncratic preference shocks generate a role for a secondary financial market: Some agents hold insufficient amounts of the liquid asset to satisfy their consumption needs while others hold too much. The financial market provides insurance against these shocks by allowing agents to rebalance their portfolio in response to these shocks, which improves the allocation of the liquid asset. ${ }^{1}$ The drawback of the financial market is that it provokes a pecuniary externality, i.e., agents do not take into account that their portfolio choice generates a general equilibrium effect on the price of the respective assets, which results in a value of the liquid asset which is too low from a societal point of view. The presence of trading frictions in the financial market can help to mitigate this externality by affecting the demand and the allocation of the liquid asset.

The fact that some trading frictions can mitigate the existing externality is not new. However, all the papers that have studied this issue so far, simply speak about "search and bargaining" taken together, and ignore the specific effects of each one of them. Indeed, a model that contains search gives rise to bilateral meetings, and when one has bilateral meetings, bargaining theory is the most obvious tool that one can use in order to study how the terms of trade within the pair are determined. But search does not necessarily imply bargaining, and even if it did, one still needs to understand which results in our theories stem from search (i.e., the difficulty to find trading partners), and which ones stem from bargaining (i.e., the fact that prices are not determined in a competitive way). To our knowledge, we are the first to disentangle the effects of search and bargaining on welfare and find that their impact is quite different.

Bargaining determines how agents split the trade surplus in the financial market. The lower the bargaining power of the liquidity demander is, the lower will be the demand for the illiquid asset in the primary market, which depresses its price and consequently also its supply due to the higher funding costs of firms. Hence, bargaining increases the demand for the liquid asset, which marginally increases its value and thereby improves the self-insurance against idiosyncratic liquidity shocks of all market participants. This effect is so strong that bargaining is welfareimproving for any inflation rate above the Friedman rule.

Search frictions determine how easy it is to find a trading partner in the financial market. Higher search frictions imply that there is a lower probability of agents to trade and therefore the illiquid asset becomes less attractive. Consequently, higher search frictions also increase the

\footnotetext{
${ }^{1}$ In this sense, the illiquid asset features "indirect liquidity", since it can be traded for the liquid asset in a secondary financial market. See Geromichalos and Herrenbrueck (2017) for a more detailed explanation.
} 
demand for the liquid asset and so its value (like in the case of bargaining). However, we show that search frictions are generally not welfare-improving. ${ }^{2}$ This is because the positive distributional effect of having more trades in the financial market dominates the negative effect on the demand for the liquid asset.

One can argue that modelling over-the-counter markets more literally requires to incorporate financial intermediaries (e.g., market makers, dealers, etc.) who bring together buyers and sellers. In such a case, the probability of meeting with a dealer could be very high, maybe even 1 , such that search frictions play a minor role. Nevertheless, the dealers would still be charging a positive bid-ask spread to investors because of the service that they provide (i.e., bringing buyers and sellers together), and that could be seen as the "bargaining" element of the model. Thus, even if there is no search friction because the investors can meet dealers at will, a secondary market organized in an over-the-counter fashion can still outperform a competitive secondary market, because the bid-ask spread that dealers charge in equilibrium has the beneficial effects described by our model. This finding has important policy implications; i.e., although policy makers can typically not control the bargaining power of investors, they can determine the structure of the market in which illiquid assets are traded. Our study shows that even in the absence of search frictions, over-the-counter markets are superior to Walrasian markets, since financial intermediaries are able to extract part of the trade surplus, which is welfare-improving due to its stimulating effect on the demand for the liquid asset.

Another innovation of our model is to endogenize the supply of the illiquid asset, which is issued in real terms. This allows us to analyze the effects of changes in monetary policy and/or the structure of the financial market on the supply side. We find that a less accommodative monetary policy; i.e., a higher inflation rate, results in a higher supply of the illiquid assets. At first, this result seems to be counterintuitive: How can it be that firms increase the supply of the illiquid asset when inflation is high? The reason behind this result is that higher inflation makes it more costly for agents to self-insure against idiosyncratic liquidity shocks by holding the liquid asset. Consequently, they increase their demand for the illiquid asset, which they can sell in the secondary financial market in case a consumption opportunity arises. Therefore, the illiquid asset exhibits a liquidity premium if inflation is high enough, which reduces the funding costs of firms and thereby leads to an increase in the total supply of the illiquid asset.

On the other hand, trading frictions in the secondary financial market reduce the demand for the illiquid asset and thereby its price. This is because due to the presence of trading frictions (search and bargaining), liquidity demanders obtain a lower share of the trade surplus and face a lower probability of finding a trading partner. In turn, a lower price of the illiquid asset increases the funding costs of firms, which is why they reduce the total supply. This result gives an intuitive explanation about why the supply of financial assets which are traded in markets that feature high search frictions (e.g. high yield corporate bonds) is generally lower than the one of financial assets which are traded in markets that feature low search frictions (e.g. high grade corporate

\footnotetext{
${ }^{2}$ As will be shown in the main text of the paper, there are a few exceptions to this statement. For instance, when the bargaining power of liquidity demanders is high and search frictions are severe, it can be locally optimal to further increase search frictions. However, this never represents a global optimum.
} 
bonds). ${ }^{3}$

\section{Literature}

Our paper is part of the "New Monetarist Economics," building on Kiyotaki and Wright (1989) and especially Lagos and Wright (2005). ${ }^{4}$ Similar to Berentsen et al. (2014), agent-types are alternating in our model, which generates a role for a financial market. Berentsen et al. (2014) analyze the benefit of search frictions in a competitive financial market. They find that increasing search frictions can be welfare-improving for high inflation rates as it incentivizes agents to increase their demand for the liquid asset. We confirm the findings of Berentsen et al. (2014), however, we also show that a competitive financial market with search frictions achieves an inferior allocation when compared with an over-the-counter market, which features bargaining. Precisely, we find that the optimal policy is to reduce the bargaining power of liquidity demanders and to reduce search frictions. This policy results in a reduction in consumption variability and achieves a superior allocation when compared with the optimal policy proposed in Berentsen et al. (2014), which increases consumption variability.

Research which is also closely related to the present paper is the study by Geromichalos and Herrenbrueck (2016a), who as well integrate an over-the-counter market in the spirit of Duffie et al. (2005) into a New Monetarist framework. The authors find that over-the-counter markets, i.e., "search and bargaining" taken together, achieve a superior allocation compared with centralized trading if inflation is low enough. We generalize the result of Geromichalos and Herrenbrueck (2016a) by disentangling the effect of search and bargaining on welfare. We show that the role of bargaining is quite different from the one of search. That is, bargaining increases the demand for the liquid asset and is therefore welfare-improving for any inflation rate above the Friedman rule. On the other hand, search frictions do not solely affect the demand but also the allocation of the liquid asset. We show that the latter effect dominates the former for a wide range of parameter values. Furthermore, we find that the optimal policy is to reduce search frictions and to reduce the bargaining power of liquidity demanders, which is true for any inflation rate above the Friedman rule. ${ }^{5}$

Along the same lines, in a model without monetary exchange, Lagos and Rocheteau (2009) explicitly incorporate the role of financial intermediaries in financial markets. Concretely, Lagos and Rocheteau (2009) assume that agents do not directly trade with each other, but that they trade with a financial intermediary who has access to a competitive interdealer market. The authors then analyze the effects of changes in trading frictions on investors' valuation over assets. They find that efficiency can only be attained if liquidity demanders obtain all the bargaining power. This is in sharp contrast to our finding, where welfare is improved by reducing the

\footnotetext{
${ }^{3}$ Other studies that also analyze the endogenous supply of illiquid assets are, for instance, Rocheteau and Rodriguez-Lopez (2014), Branch et al. (2016), and Geromichalos and Herrenbrueck (2016b).

${ }^{4}$ For an overview of this literature, see Williamson and Wright (2010), Nosal and Rocheteau (2011), and Lagos et al. (2015).

${ }^{5}$ We present more details of Berentsen et al. (2014) and Gerimochalos and Herrenbrueck (2016a) and compare these studies to our model in Section 10.
} 
bargaining power of liquidity demanders. The reason for this difference is that we incorporate monetary exchange, while Lagos and Rocheteau (2009) do not. This gives rise to the presence of a pecuniary externality, which causes the equilibrium to be inefficient. Since in a such an environment the First Welfare Theorem does not go through, it is the case that some frictions are actually welfare-improving.

In a similar model that incorporates monetary exchange, Lagos and Zhang (2015) confirm the finding of Lagos and Rocheteau (2009) that a lower bargaining power of liquidity demanders results in lower asset prices. However, the authors do not focus on the social implications of such a shift in bargaining power, while we do. This allows us to show that a reduction in the bargaining power of liquidity demanders does not only reduce asset prices, but does also improve welfare. Furthermore, Lagos and Zhang (2015) mainly focus on asset pricing, while we analyze the social role of trading frictions in financial markets.

Our paper is also related to the literature that studies the markets for risk-sharing by means of insurance contracts (e.g., Parlour and Rajan 2001, Thompson 2010, Duffie and Zhu 2011, Leitner 2013, Acharya and Bisin 2014, and Stephens and Thompson 2014). These papers are mainly concerned about the moral hazard of the involved counterparties in the insurance contract and how over-the-counter markets affect their behavior. Moral hazard arises, because agents do not need to disclose trade information publicly in over-the-counter markets. In contrast, in centralized markets this information is revealed, thereby preventing moral hazard (see Leitner 2013, and Acharya and Bisin 2014). Duffie and Zhu (2011) and Stephens and Thompson (2014) also analyze the benefits of centralized markets. In contrast to the previous studies, their focus is on the netting of positions and pooling of risk through centralized markets. Duffie and Zhu (2011) show that centralized trading can be beneficial due to the netting of trades, which reduces counterparty risk. In contrast, Stephens and Thompson (2014) show that the pooling of risk through centralized markets results in an adverse selection of insurance providers, as everybody wants to benefit from the low insurance premia of bad providers. Our study differs from this literature, because neither counterparty risk nor asymmetric information exists among agents. We show that a centralization of financial markets is welfare-decreasing, since it reduces the incentive of agents to self-insure against idiosyncratic liquidity shocks.

\section{Environment}

Time is discrete and the economy is populated by a $[0,1]$-continuum of infinitely lived agents. In each period, there are three markets that open sequentially. In the first market, agents trade liquid for illiquid assets. In the second market, agents produce or consume market-2 goods. In the third market, financial contracts are redeemed and agents produce and consume market-3 goods. We refer to these three markets as the financial market, the goods market, and the centralized market.

At the beginning of each period, agents receive an idiosyncratic i.i.d. preference shock that determines the agent's ability to produce or consume the market-2 good in the goods market: An agent can produce but not consume with probability $n$, while he can consume but not produce 
with probability $1-n$. In the goods market, trading is competitive. Consumers enjoy utility $u(q)$ from $q$ consumption, where $u^{\prime}(q),-u^{\prime \prime}(q)>0, u^{\prime}(0)=\infty$, and $u^{\prime}(\infty)=0$. Producers incur a utility cost $c(q)=q$ from producing $q$ units of the market-2 good. No record-keeping technology exists and agents cannot commit. As a consequence, producers ask for immediate compensation from consumers; i.e., a medium of exchange is required.

The centralized market is frictionless, and agents can produce and consume the market-3 good. Agents receive utility $U(x)$ from $x$ consumption, where $U^{\prime}(x),-U^{\prime \prime}(x)>0, U^{\prime}(0)=\infty$, and $U^{\prime}(\infty)=0$. The market-3 good is produced by means of a linear production technology; i.e., $h$ hours of work produce $h$ units of the market-3 good. Agents discount between two consecutive periods with the discount factor $\beta \in(0,1)$. In this third market, a central bank supplies the liquid asset: A perfectly divisible, storable, and intrinsically useless object called money. The change in the stock of money is given by $M_{t+1}=\gamma M_{t}$, where $\gamma$ denotes the gross growth rate of money and $M_{t}\left(M_{t+1}\right)$ denotes the stock of money in period $t(t+1)$. The central bank injects (withdraws) money through a lump-sum transfer $T_{t}$ to all agents, where $T_{t}=M_{t+1}-M_{t}=(\gamma-1) M_{t}$. For notational simplicity, we omit the time subscript $t$ going forward and denote next-period variables by +1 , and previous-period variables by -1 .

Our model assumes a continuum $\ell>0$ of homogeneous firms which issue the illiquid asset in the centralized market: A storable and perfectly divisible object called bond. ${ }^{6}$ In contrast to money, bonds are intangible objects; i.e., no physical object exists. As a consequence, only money can serve as a medium of exchange in the goods market. ${ }^{7}$ Any firm can enter the market, but there is an entry cost $C$ in terms of market-3 goods. A generic firm issues a quantity of $\mathfrak{b}$ private bonds at price $\rho \leq \gamma$ to finance its project. Bonds can only be purchased by market-3 goods, since firms use these goods as an input for their projects; i.e., bonds are issued in real terms. If the project is successful during the period, then the firm honors its obligation and pays back 1 unit of the market-3 good in the next period's centralized market. If a firm cannot repay its obligation, then the firm defaults and will be forced permanently out of the market. The period payoff of a generic firm is $z f(\rho \mathfrak{b}) / \ell$, where $f^{\prime}>0, f^{\prime \prime}<0, f(0)=0, f^{\prime}(0)=\infty$, and $z \in[0,1] .{ }^{8}$ A firm's project is fully successful with probability $1-g(z=1)$ and it is not successful with probability $g(z=0)$. A project's payoff $z$ is realized before the next period's centralized market opens, but after the financial market closes. Firms cannot issue more bonds than $\overline{\mathrm{b}}$, where $f(\rho \overline{\mathrm{b}})=\ell \overline{\mathrm{b}}$, since it is certain that bonds cannot be redeemed for $\mathbb{b}>\overline{\mathrm{b}}$ for any realization of $z$.

At the beginning of each period, and after the realization of the idiosyncratic preference shock, agents can trade money for bonds in the financial market. Consumers and producers meet at

\footnotetext{
${ }^{6}$ Our firms have some similarities to the ones studied in Mortensen and Pissaridies (1994), Williamson (1987, 2012), and Berentsen et al. (2011). However, we deviate in several dimensions from these studies, as will be shown further on in the paper.

${ }^{7}$ An extended discussion about the frictions that make money essential can be found, for instance, in Kocherlakota (1998), Wallace (2001), Lagos and Wright (2005) and Shi (2006). A more detailed explanation about why bonds cannot serve as a medium of exchange is provided by Kocherlakota (2003), Andolfatto (2011), Berentsen and Waller (2011), and Berentsen et al. (2014 and 2016).

${ }^{8}$ The assumption that the period payoff of a generic firm is decreasing in the number of firms in the market is needed to endogenize $\ell$ through the free entry condition.
} 
random in bilateral meetings according to a reduced-form matching function $\xi \mathcal{M}(n, 1-n)$, where the scaling parameter $\xi$ determines the efficiency of the matching process (see e.g., Rocheteau and Weill 2011). We assume that the matching function has constant returns to scale, and is continuous and increasing with respect to each of its arguments. A consumer meets a producer with probability $\delta=\xi \mathcal{M}(n, 1-n)(1-n)^{-1}$, and a producer meets a consumer with probability $\delta^{p}=\xi \mathcal{M}(n, 1-n) n^{-1}=\delta(1-n) n^{-1}$. Once in a match, agents bargain over the quantity of money and bonds to be exchanged. Agents who are able to trade in this market are called active and those who are not are called passive.

\section{The Agent's Decisions}

Hereafter, we focus on the agent's decision problem in a representative period $t$. We first present the last market (centralized market) and work backwards to the first market (financial market).

\subsection{Centralized Market}

In the centralized market, agents can produce and consume the market-3 good and receive the lump-sum money transfer $T$ from the central bank. Old and new firms issue bonds at price $\rho \leq \gamma$ in this market, and agents decide on the amount of money and bonds that they will take into the next period. An agent entering the centralized market with $m$ units of money and $b$ units of bonds has the value function $V_{3}(m, b)$ and solves the following decision problem:

$$
V_{3}(m, b)=\max _{x, h, m_{+1}, b_{+1}}\left[U(x)-h+\beta V_{1}\left(m_{+1}, b_{+1}\right)\right]
$$

subject to

$$
x+\phi m_{+1}+\rho b_{+1}=h+\phi m+(1-g) b+\phi T,
$$

where $\phi$ denotes the price of money in terms of market-3 goods, and $h$ denotes the hours worked. Due to the linear production technology, the choice of $m_{+1}$ and $b_{+1}$ is independent of $m$ and $b$. As a result, the distribution of $m$ and $b$ is degenerate at the end of a period and each agent exits the market with the same amount of money and bonds. Furthermore, all agents buy the same amount of bonds from each firm; i.e., all agents hold an identical fraction of defaulting bonds in their portfolios. ${ }^{9}$

\subsection{Goods Market}

In the goods market, all agents take prices as given and trade against the market. A producer entering the goods market has the following decision problem:

\footnotetext{
${ }^{9}$ To see this, consider the following example: $\mathfrak{b}=9$ and $\ell=10 / 9$ according to decisions of the firms. Then, we have $\ell \mathrm{b}=b=10$; i.e., each firm offers 9 bonds, but each agent holds 10 bonds. In other words, agents must be able to buy the same number of bonds from each firm.
} 


$$
V_{2}^{p}(m, b)=\max _{q_{p}}\left[-c\left(q_{p}\right)+V_{3}\left(m+p q_{p}, b\right)\right],
$$

where $V_{2}^{p}(m, b)$ denotes his value function, $p$ denotes the price of one unit of the market-2 good $q$, and $q_{p}$ satisfies the market clearing condition

$$
(1-n)[\delta \hat{q}+(1-\delta) q]=n q_{p}
$$

In equilibrium, active consumers will hold more money than passive consumers and are therefore able to consume more in this market. This is because active consumers had the possibility to rebalance their portfolio in the financial market, while passive consumers did not. Quantities associated with active agents are denoted with a hat $\left(^{\wedge}\right)$. The market clearing condition (4) states that the produced quantity of a consumer, $q_{p}$, equals the sum of the quantities consumed by active consumers, $(1-n) \delta \hat{q}$, and by passive consumers, $(1-n)(1-\delta) q$, divided by the number of producers in the market, $n$. Due to the assumption of competitive pricing, it is easy to show that producers do not earn any surplus; i.e., that the marginal cost of producing $q_{p}$ units of market-2 goods equals the market price, $c^{\prime}\left(q_{p}\right)=1=p \phi$.

A consumer entering the goods market has the following decision problem:

$$
V_{2}^{c}(m, b)=\max _{q}\left[\begin{array}{c}
u(q)+V_{3}(m-p q, b) \\
\text { s.t. } m \geq p q .
\end{array}\right],
$$

where $V_{2}^{c}(m, b)$ denotes his value function. The budget constraint simply states that a consumer cannot spend more money than he has.

If the budget constraint is non-binding, then we have $\partial q / \partial m=0$, and consequently consumers are able to consume the efficient quantity $q^{*}$, which is the solution of $u^{\prime}\left(q^{*}\right)=1$. If the constraint is binding, then we have $\partial q / \partial m=\phi$ and $u^{\prime}(q)>1$, where we have used $1=p \phi$. Hence, consumers spend all their money and consumption is inefficiently low.

\subsection{Financial Market}

In the financial market, consumers and producers meet at random in bilateral meetings, and the trade surplus is split according to the Kalai (1977) solution. The Kalai trading protocol has the property of being strongly monotonic; i.e., no agent is made worse off by an expansion of the bargaining set. ${ }^{10}$ An agent enters the financial market with a portfolio $\left(m_{j}, b_{j}\right)$ and leaves it with a portfolio $\left(\hat{m}_{j}, \hat{b}_{j}\right)$, where $j=c$ for consumers, and $j=p$ for producers. ${ }^{11}$ By the market

\footnotetext{
${ }^{10}$ For a formalization of Kalai bargaining, see Rocheteau and Wright (2005), and Aruoba et al. (2007). Recent studies that apply Kalai bargaining to financial markets are, for example, Geromichalos and Herrenbrueck (2016a), and Berentsen et al. (2016). A study of the monotonicity properties of Kalai can be found in Chun and Thomson (1988).

${ }^{11}$ Note that consumers and producers leave the centralized market with the same portfolio, therefore $m_{c}=m_{p}=$ $m$ and $b_{c}=b_{p}=b$. We just use the subscripts to differentiate consumers from producers more easily.
} 
clearing condition, we obtain

$$
\hat{m}_{c}-m_{c}=-\left(\hat{m}_{p}-m_{p}\right) \quad \text { and } \quad \hat{b}_{p}-b_{p}=-\left(\hat{b}_{c}-b_{c}\right) .
$$

An active producer spends $d_{m} \equiv \hat{m}_{c}-m_{c}$ units of money and an active consumer sells $d_{b} \equiv \hat{b}_{p}-b_{p}$ units of bonds, which implies that the budget constraints of a producer and a consumer are given by

$$
\phi m_{p} \geq \phi d_{m} \quad \text { and } \quad b_{c} \geq d_{b} .
$$

The total trade surplus in this market is $u(\hat{q})-u(q)-\phi d_{m}$, where $u(\hat{q})-u(q)-(1-g) d_{b}$ is the consumer's surplus, and $(1-g) d_{b}-\phi d_{m}$ is the producer's surplus. The Kalai constraint splits the total trade surplus among matched agents according to their bargaining power

$$
(1-\eta)\left[u(\hat{q})-u(q)-(1-g) d_{b}\right]=\eta\left[(1-g) d_{b}-\phi d_{m}\right],
$$

where $\eta$ is the bargaining power of a consumer, and $1-\eta$ that of a producer. An active agent's decision problem is

$$
K\left(m_{c}, m_{p}, b_{c}, b_{p}\right) \equiv \max _{d_{m}, d_{b}}\left[u(\hat{q})-u(q)-\phi d_{m}\right] \quad \text { s.t. } \quad(6) \text { and }(7) .
$$

That is, the Kalai protocol maximizes the total trade surplus and is therefore efficient. If $K\left(m_{c}, m_{p}, b_{c}, b_{p}\right)$ is differentiable with respect to $x=m_{c}, m_{p}, b_{c}, b_{p}$, then we have

$$
\frac{\partial K}{\partial x}=u^{\prime}(\hat{q}) \frac{\partial \hat{q}}{\partial x}-u^{\prime}(q) \frac{\partial q}{\partial x}-\phi \frac{\partial d_{m}}{\partial x} .
$$

If the active agent's budget constraints (6) are non-binding, then the first-order condition of (8) with respect to $d_{m}$ is $u^{\prime}(\hat{q})=1$; i.e., active consumers can consume the efficient quantity. The value functions of consumers and producers entering the financial market are given by

$$
\begin{aligned}
V_{1}^{c}\left(m_{c}, b_{c}\right) & =\delta \eta K\left(m_{c}, m, b_{c}, b\right)+V_{2}^{c}\left(m_{c}, b_{c}\right), \\
V_{1}^{p}\left(m_{p}, b_{p}\right) & =\delta_{p}(1-\eta) K\left(m, m_{p}, b, b_{p}\right)+V_{2}^{p}\left(m_{p}, b_{p}\right),
\end{aligned}
$$

where $(m, b)$ denotes the portfolio of the trading partner.

\section{The Firm's Decisions}

Firms only interact with agents in the centralized market. If their project was successful during the period, they honor their obligation and repay 1 unit of the market-3 good for each maturing bond. Furthermore, old firms which were successful in the previous period and new firms which can afford to pay the entry cost $C$ in terms of market-3 goods issue a quantity $\mathfrak{b}$ of corporate bonds at price $\rho \leq \gamma$ to finance their new projects. A firm has the value function $V_{f}$ and solves 
the following decision problem

$$
V_{f}=\max _{\mathfrak{b} \in[0, \bar{b}]}(1-g)\left[\frac{f(\rho \mathfrak{b})}{\ell}-\mathfrak{b}+\beta V_{f+}\right],
$$

where $\ell>0$ denotes the measure of firms in the market, and $f(\rho \mathrm{b}) / \ell$ denotes the period payoff of a generic firm. A firm with a project realization of $z=0$ (probability $g$ ) is not able to honor its debt and will consequently be forced to default and to be permanently excluded from the market. A firm with a project realization of $z=1$ (probability $1-g$ ) can fulfill its obligations $\mathbb{b}$ and has the continuation value $\beta V_{f+}$. The firm's decision problem depends on monetary policy through the issue price of bonds in the centralized market, $\rho$, which affects the number of firms in the market, $\ell$, and the real quantity of bonds issued by a generic firm, $\mathfrak{b}$.

In the steady state, it holds that $V_{f+}=V_{f}$ and thus, we can simplify a firm's value function to

$$
V_{f}=\frac{1-g}{1-\beta(1-g)}\left[\frac{f(\rho \mathfrak{b})}{\ell}-\mathbb{b}\right] .
$$

From the above equation, it is easy to see that the optimal real quantity of bonds issued by a firm, b, satisfies

$$
\rho f^{\prime}(\rho \mathfrak{b})=\ell \text {. }
$$

Furthermore, by the entry condition, we also have $V_{f}=C$ in equilibrium. Hence, a firm will not strategically default if $\beta C \geq \mathfrak{b}$, or equivalently if

$$
\beta C=\frac{\beta(1-g)}{1-\beta(1-g)}\left[\frac{f(\rho \mathfrak{b})}{\ell}-\mathfrak{b}\right] \geq \mathfrak{b} .
$$

Equation (11) and (12) state that the number of firms in the market, $\ell$, and the real quantity of bonds issued by a firm, $\mathfrak{b}$, are both completely determined by the endogenous price of bonds in the centralized market, $\rho$, the production technology of firms, $f(x)$, and the exogenous terms $C, \beta$, and $g$. By the resource constraint, it also holds in equilibrium that $b=\ell \mathfrak{b}$; i.e., that the total real quantity of bonds held by agents, $b$, equals the total real quantity of bonds issued by all firms, $\ell$ b.

\section{Equilibria}

Hereafter, we characterize two stationary monetary equilibria, where all agents follow identical strategies and where real variables are constant over time. In the first equilibrium, trading is unconstrained; i.e., (6) is non-binding. We label this equilibrium as type-A. In the second equilibrium, active producers are unconstrained, while the bond constraint of active consumers is binding. This equilibrium is labeled as type-B. All proofs are relegated to the Appendix. 


\subsection{Type-A Equilibrium}

In a type-A equilibrium, the bond constraint of active consumers is non-binding, $b_{c}>d_{b}$, which allows them to consume the efficient quantity, $u^{\prime}(\hat{q})=1$. Furthermore, the cash constraint of active producers is non-binding, $\phi m_{p}>\phi d_{m}$, which means that they are indifferent between holding money or bonds.

Proposition 1 A type-A equilibrium is a list $\left\{\hat{q}, q, q_{p}, \rho, \mathfrak{b}, \ell\right\}$ satisfying (4), (11), (12), and

$$
\begin{aligned}
1 & =u^{\prime}(\hat{q}), \\
\frac{\gamma}{\beta} & =(1-n) \delta \eta\left[u^{\prime}(\hat{q})-u^{\prime}(q)\right]+(1-n) u^{\prime}(q)+n, \\
\rho & =\beta(1-g) .
\end{aligned}
$$

Equation (13) states that active consumers can consume the efficient quantity, because the budget constraints (6) are non-binding in the financial market. Equation (14) states that the marginal benefit of acquiring one additional unit of money equals the sum of three terms: If an agent turns out to be a consumer (active or passive), he has the marginal utility $u^{\prime}(q)$; if an agent turns out to be an active consumer, he is able to consume the efficient quantity and consequently his marginal utility is lower than for a passive consumer, $\eta\left[u^{\prime}(\hat{q})-u^{\prime}(q)\right]<0$; if an agent turns out to be a producer (active or passive), he obtains a marginal utility of 1 . Equation (15) states that bonds are priced at their fundamental value, $\rho=\beta(1-g)$.

\subsection{Type-B Equilibrium}

In a type-B equilibrium, the bond constraint of active consumers is binding, $b_{c}=d_{b}$; i.e., they would like to sell more bonds than they have in order to satisfy their consumption needs. Hence, they cannot achieve the efficient quantity and $u^{\prime}(\hat{q})>1$ holds. As in the type-A equilibrium, the cash constraint of active producers is non-binding, $\phi m_{p}>\phi d_{m}$.

Proposition 2 A type-B equilibrium is a list $\left\{\hat{q}, q, q_{p}, \rho, \mathfrak{b}, \ell\right\}$ satisfying (4), (11), (12), and

$$
\begin{aligned}
(1-g) \ell \vec{b} & =(1-\eta)[u(\hat{q})-u(q)]+\eta(\hat{q}-q), \\
\frac{\gamma}{\beta} & =(1-n)\left[\delta u^{\prime}(\hat{q}) \frac{\eta+(1-\eta) u^{\prime}(q)}{\eta+(1-\eta) u^{\prime}(\hat{q})}+(1-\delta) u^{\prime}(q)\right]+n \\
\rho & =\beta(1-g)\left[1+(1-n) \delta \eta \frac{u^{\prime}(\hat{q})-1}{\eta+(1-\eta) u^{\prime}(\hat{q})}\right] .
\end{aligned}
$$

Equation (16) is based on the Kalai constraint, where we used $b=\ell \mathfrak{b}$. Equation (17) states that the marginal benefit of acquiring one additional unit of money equals 1 for producers (active or passive), it equals the marginal utility $u^{\prime}(q)$ for passive consumers, and it equals the share of the surplus $u^{\prime}(\hat{q})\left[\eta+(1-\eta) u^{\prime}(q)\right]\left[\eta+(1-\eta) u^{\prime}(\hat{q})\right]^{-1}$ for active consumers. Equation (18) states that bonds are priced above their fundamental value, $\beta(1-g)$, and exhibit a liquidity premium 
equal to $(1-n) \delta \eta\left[u^{\prime}(\hat{q})-1\right]\left[\eta+(1-\eta) u^{\prime}(\hat{q})\right]^{-1}$. The equation shows that the liquidity premium is affected by $\gamma$ through $u^{\prime}(\hat{q})$, and by the trading frictions $\delta$ and $\eta$. Compared with the type-A equilibrium, $\rho$ is higher because (i) $u^{\prime}(\hat{q})>1$ and because (ii) lower trading frictions (higher values of $\delta$ and $\eta$ ) also lead to an increase in the liquidity premium. ${ }^{12}$

\section{Regions of Existence}

Hereafter, we specify two non-overlapping regions in which the type-A and the type-B equilibria exist, as a function of the inflation rate $\gamma$ and the entry cost $C$. In order to do this, we first need to derive the reaction of firms in equilibrium. Let $\alpha(x)=-x f^{\prime \prime}(x) / f^{\prime}(x)$ denote the coefficient of risk aversion of $f(x)$, and let $\epsilon(x)=x f^{\prime}(x) / f(x)$ denote the elasticity of $f(x)$. In equilibrium, firms' decisions are completely determined by the endogenous bond price $\rho$ and the exogenous terms $C, g$, and $\beta$ through (11) and (12). In Lemma 3, we derive the effect on the real quantity of bonds issued by a generic firm, $\mathfrak{b}$, the number of firms in the market, $\ell$, and the total real quantity of bonds issued, $\ell \mathfrak{b}=b$, in response to a marginal increase in the exogenous terms $C$, $g$, and $\beta$.

Lemma 3 In equilibrium,

$$
\begin{array}{lll}
\frac{\partial \mathfrak{b}}{\partial C}>0, & \frac{\partial \mathfrak{b}}{\partial g}>0, & \frac{\partial \mathfrak{b}}{\partial \beta}<0, \\
\frac{\partial \ell}{\partial C}<0, & \frac{\partial \ell}{\partial g}<0, & \frac{\partial \ell}{\partial \beta}>0
\end{array}
$$

and

$$
\frac{\partial \ell \mathfrak{b}}{\partial C}, \frac{\partial \ell \mathfrak{b}}{\partial g},-\frac{\partial \ell \mathfrak{b}}{\partial \beta} \begin{cases}<0, & \text { if } \alpha(\rho \mathfrak{b})>1 \\ =0, & \text { if } \alpha(\rho \mathfrak{b})=1 \\ >0, & \text { if } \alpha(\rho \mathrm{b})<1\end{cases}
$$

Lemma 3 states that the response of the total real quantity of bonds issued, $\ell \mathfrak{b}=b$, to a marginal increase in the exogenous terms $C, g$, and $\beta$, critically depends on the coefficient of risk aversion of $f(x)$. For instance, a standard production function of the form $f(x)=A x^{1-\alpha}$ with $1-\alpha \leq \beta(1-g), \alpha<1$, and $A>0$ satisfies $\alpha(x)=\alpha<1$, which implies that the total real quantity of bonds, $\ell \mathbb{b}=b$, is increasing in $C, g$, and $\beta$. It is increasing in $C$, since a higher entry cost prevents more firms to enter the market and so increases the period payoff of the remaining once, which results in an increase in the total issuance. A higher default probability $g$ reduces the continuation value of firms, which forces some firms out of the market and thus also increases the period payoff of the remaining once and so the total issuance. An increase in $\beta$ lowers the funding costs of firms, which results in more firms in the market. Although the period payoff of each firm decreases, the total issuance increases.

\footnotetext{
${ }^{12}$ See Geromichalos and Herrenbrueck (2016a) for a more detailed discussion about the conditions which must be satisfied for a liquidity premium to exist in the centralized market.
} 
In Lemma 4 , we derive the effect on $\mathfrak{b}, \ell$, and $\ell \mathfrak{b}=b$, in response to a marginal increase in the endogenous bond price $\rho$.

Lemma 4 In equilibrium,

$$
\frac{\partial \mathfrak{b}}{\partial \rho} \begin{cases}<0, & \text { if } \alpha(\rho \mathfrak{b})>1-\epsilon(\rho \mathbb{b}), \\ =0, & \text { if } \alpha(\rho \mathfrak{b})=1-\epsilon(\rho \mathbb{b}), \\ >0, & \text { if } \alpha(\rho \mathbb{b})<1-\epsilon(\rho \mathbb{b}), \\ & \frac{\partial \ell}{\partial \rho}>0,\end{cases}
$$

and

$$
\frac{\partial \ell \mathfrak{b}}{\partial \rho} \begin{cases}<0, & \text { if } \alpha(\rho \mathfrak{b})>1 \\ =0, & \text { if } \alpha(\rho \mathfrak{b})=1 \\ >0, & \text { if } \alpha(\rho \mathfrak{b})<1 .\end{cases}
$$

Note, that the incentive constraint

$$
\frac{\beta(1-g)}{1-\beta(1-g)}\left[\frac{f(\rho \mathfrak{b})}{\ell}-\mathfrak{b}\right] \geq \mathbb{b}
$$

is satisfied if, and only if,

$$
\epsilon(\rho \mathfrak{b}) \equiv \frac{f^{\prime}(\rho \mathfrak{b}) \rho \mathfrak{b}}{f(\rho \mathfrak{b})} \leq \beta(1-g)<1 ;
$$

i.e., the elasticity of $f(\rho \mathrm{b})$ must be bounded above by $\beta(1-g)$ in equilibrium. Using the same example of a standard production function from above, we obtain $\epsilon(x)=1-\alpha<1$ and hence $\alpha(x)=1-\epsilon(x)$; i.e., an increase in the endogenous bond price $\rho$ has no effect on $\mathfrak{b}$. However, since $\alpha(x)=\alpha<1$, the total issuance of bonds increases when $\rho$ increases, since more firms enter the market due to the lower funding costs.

With the results from Lemma 3 and Lemma 4, we are able to derive the regions of existence of the type-A and the type-B equilibrium. In Proposition 5, we derive these regions for an economy where the entry cost is relatively low, $C<\bar{C}$, and where the production technology $f(x)$ satisfies $\alpha(x)=-x f^{\prime \prime}(x) / f^{\prime}(x)<1$ and $\epsilon(x)=x f^{\prime}(x) / f(x) \leq \beta(1-g)$ for all $x \geq 0$.

Proposition 5 Let $\alpha(x)<1$ and $\epsilon(x) \leq \beta(1-g)$ for all $x>0$. There exists a $\bar{C}$ such that for any $C<\bar{C}$ we can find $\gamma_{A B} \equiv \gamma_{A B, C}$ so that, if $\gamma<\gamma_{A B}$, then equilibrium prices and quantities are characterized by Proposition 1; and if $\gamma>\gamma_{A B}$, then equilibrium prices and quantities are characterized by Proposition 2.

While $\alpha(x)<1$ and $\epsilon(x) \leq \beta(1-g)$ is satisfied by many standard production functions, the assumption of $C<\bar{C}$ requires more explanation. We use this assumption to assure that an equilibrium where money is scarce and bonds are plentiful does not exist. We discuss this equilibrium in more detail in Section 10. 


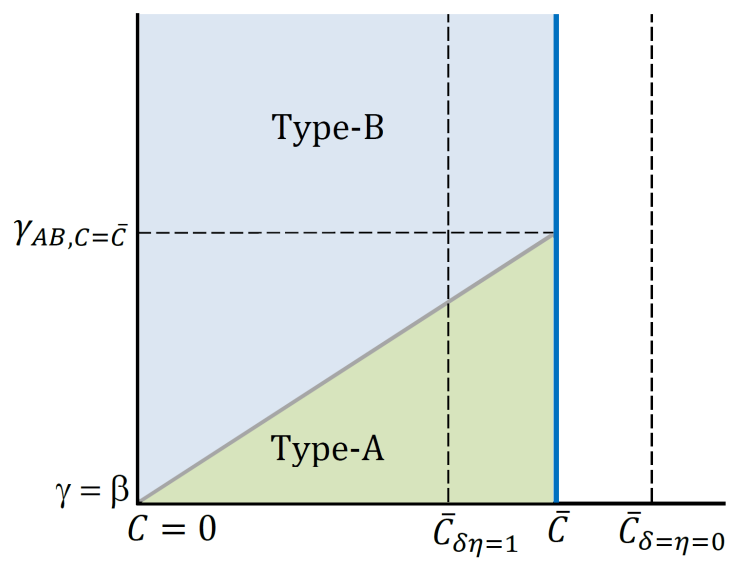

Figure 1: Regions of EXISTEnCE FOR $\delta \eta<1$

Proposition 5 states that for low inflation rates, $\gamma<\gamma_{A B}$, the type-A equilibrium exists, where trading is unconstrained. For higher inflation rates, active consumers need to sell a larger fraction of their bond holdings in order to satisfy their consumption needs. Thus, for $\gamma>\gamma_{A B}$ the constraint on bond holdings becomes binding and the type-B equilibrium exists. For ease of understanding, Figure 1 shows the regions of existence as a function of the inflation rate $\gamma$ and the entry cost $C$. The chart shows the respective regions for $0<\delta \eta<1$. In the proof of Proposition 5, we show that at $C=\bar{C}$, the critical inflation rate that separates the type-A from the type-B equilibrium equals

$$
\gamma_{A B, C=\bar{C}}=\beta\left\{(1-n)\left[\delta \eta+(1-\delta \eta) u^{\prime}\left(q^{*} / 2\right)\right]+n\right\} .
$$

From the above equation, it is clear that for $\delta \eta=1$ we obtain $\gamma_{A B, C=\bar{C}}=\beta$, and hence for any $C<\bar{C}_{\delta \eta=1}$, only the type-B equilibrium exists for $\gamma>\beta$ when $\delta \eta=1$. Furthermore, we find that $\bar{C}$ is decreasing in $\eta$ and independent of $\delta$; i.e., for $\delta \eta=1$, we obtain a lower value of $\bar{C}$ as compared to $0<\delta \eta<1$ (see Figure 1 ).

\section{The Role of Trading Frictions}

In this section, we analyze the social benefits of trading frictions in financial markets. In order to this, we first derive our model for a centralized financial market and then analyze under what conditions an over-the-counter market achieves a superior allocation. A centralized market differs from the above-analyzed over-the-counter market in two dimensions. First, there is no random matching and thus agents trade with certainty. Second, there is no bargaining, since agents trade against the market, and the market price equates aggregate supply and aggregate demand. To make the results comparable to the above analysis, we assume in a first step that agents enter the centralized market probabilistically with the probabilities $\delta$ and $\delta^{p}$, respectively. This allows 
us to compare the implications of the different market structures, since the number of trades are identical with this assumption.

We now derive the type-A and the type-B equilibrium for a centralized financial market with probabilistic entry, which is shown by Proposition 6 .

Proposition 6 Equilibrium prices and quantities characterized by Proposition 1 and 2 coincide with competitive pricing in the financial market if, and only if, $\eta=1$.

Proposition 6 states that a centralized financial market with probabilistic entry coincides with an over-the-counter market under the condition that consumers obtain the entire trade surplus $(\eta=1)$. In a first step, we thus analyze the effects of increasing $\eta$ in the over-the-counter market in the type- $\mathrm{A}$ and the type-B equilibrium.

In Figure 2, we show the regions of existence in terms of search frictions $(\delta)$ and the bargaining power of consumers $(\eta)$ for $C<\bar{C}_{\delta \eta=1}$. To draw this figure, we assume that the economy is initially characterized by $\delta=\eta=0.5$ and that the entry cost $C$ is sufficiently large, such that the type-A equilibrium is supported for $\delta=\eta=0 .{ }^{13}$ The figure shows that the economy is initially in the type-B equilibrium with $\gamma$ being close to $\gamma_{A B}$, which is visible by the small distance to the type-A equilibrium. Increasing $\eta$ from $\eta=0.5$ to $\eta=1$ moves the economy to the right in Figure 2 ; i.e., it reduces $\gamma_{A B, C=\bar{C}}$, which is clearly formulated in (19), where we obtain $\partial \gamma_{A B, C=\bar{C}} / \partial \eta<0$. Furthermore, the figure shows that the type-B equilibrium is supported for large values of $\delta$ and $\eta$. To see this, note that by (14) we know that in the type-A equilibrium it holds that

$$
u^{\prime}(q)=\frac{\gamma-\beta[(1-n) \delta \eta+n]}{\beta(1-n)(1-\delta \eta)}
$$

so $q$ is close to $\hat{q}$ when $\gamma$ is close to $\beta$, since $u^{\prime}(\hat{q})=1$ by (13). For a given $\gamma$, the right-hand side of the above equation grows infinitely large, as $\delta \eta$ approaches the value one. Since the left-hand side of the equation is bounded above by $u^{\prime}\left(q^{*} / 2\right)$, the type-A equilibrium no longer exists for large $\delta \eta{ }^{14}$

In order to analyze the social implication of increasing $\eta$ in the type-B equilibrium, we have to define the welfare function, which is given by

$$
(1-\beta) \mathcal{W} \equiv U\left(x^{*}\right)-x^{*}+(1-n)[\delta u(\hat{q})+(1-\delta) u(q)]-n q_{p} .
$$

The term $U\left(x^{*}\right)-x^{*}$ denotes an agent's expected utility in the centralized market. It is immediate from the first-order condition in the centralized market with repsect to $x$ that every agent consumes the efficient quantity $x^{*}$, independent of his trading history. The term $(1-n)[\delta u(\hat{q})+(1-\delta) u(q)]$ denotes an agent's expected utility in the goods market if he becomes a consumer; and the term $-n q_{p}$ denotes an agent's expected disutility if he becomes a producer in the goods market.

\footnotetext{
${ }^{13}$ This condition is satisfied for a wide range of parameter values and functional forms of the utility- and production functions.

${ }^{14}$ Note that $q \geq q^{*} / 2$ holds in the type-A equilibrium and $u^{\prime}$ is a decreasing function.
} 


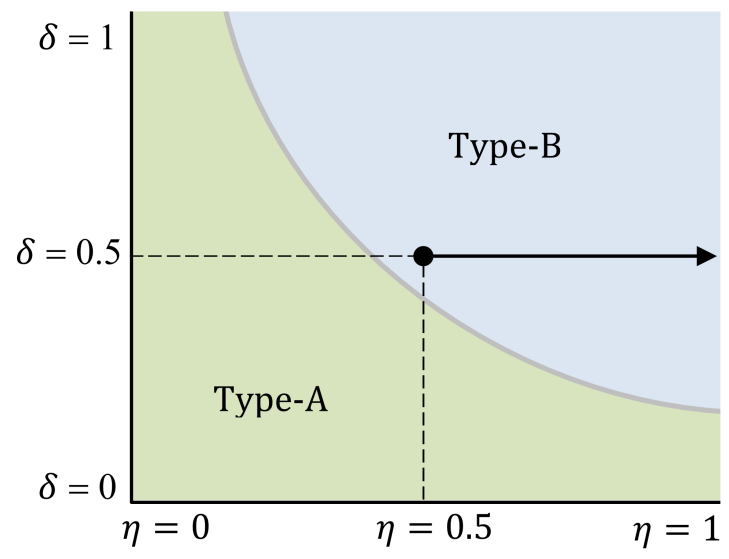

Figure 2: INCREASING $\eta$ IN THE TYPE-B EQUILIBRIUM

Proposition 7 summarizes the effects of an increase in $\eta$ in the type-B equilibrium; i.e., a shift in bargaining power that coincides with a competitive financial market for $\eta=1$.

Proposition 7 Let $\delta$ and $\eta$ be large in the type-B equilibrium. Then, we have

$$
\frac{\partial \rho}{\partial \eta}>0, \quad \frac{\partial(\ell \mathbb{b})}{\partial \eta}>0, \quad \frac{\partial \hat{q}}{\partial \eta}<0, \quad \frac{\partial q}{\partial \eta}<0, \quad \frac{\partial \mathcal{W}}{\partial \eta}<0 .
$$

Proposition 7 states that increasing the consumer's bargaining power $\eta$ results in a higher liquidity premium on bonds in the centralized market; i.e., $\rho$ increases. This is because active consumers obtain a larger fraction of the trade surplus in the financial market, which makes bonds more valuable to them such that their demand for bonds increases. This effect is so strong that it dominates the effect of a larger supply issued by firms who want to benefit from the lower funding costs. Due to the larger supply of bonds, there is a higher trading volume in the financial market, even though the number of matches $\delta(1-n)$ remains unchanged. The drawback of an increase in $\eta$ is that the demand for money decreases. Consequently, the value of money in terms of goods decreases, $\phi m=q$, and passive consumers consume less in the goods market. The decrease in the value of money is so strong, that also active consumers consume less, although the trading volume in the financial market increases. Hence, active and passive agents consume less in the goods market in response to an increase in $\eta$, which is welfare-decreasing. These results hold for any positive inflation rate, $\gamma>\beta$, and large values of $\delta$ and $\eta$.

We now analyze whether $\partial \mathcal{W} / \partial \eta<0$ also holds for smaller values of $\delta$ and $\eta$, where the type-B equilibrium is close to the type-A equilibrium, which is shown by Corollary 8.

Corollary 8 Consider the type- $B$ equilibrium, given $\gamma, \delta$, and $\eta$. If this type- $B$ equilibrium is close to the type- $A$ equilibrium, we have $\partial \mathcal{W} / \partial \eta<0$. 
Corollary 8 confirms that increasing $\eta$ is also welfare-decreasing for smaller values of $\delta$ and $\eta$ in the type-B equilibrium.

In a next step, we analyze an economy which features high search frictions (low $\delta$ ), such that $\gamma<\gamma_{A B}$ for $\eta=0.5$ and $C<\bar{C}_{\delta \eta=1}$. In contrast to the previous analysis, an increase in the bargaining power of consumers to $\eta=1$ keeps the economy in the type-A equilibrium. Figure 3 visualizes this shift in bargaining power using the black arrow. Figure 3 shows that increasing $\eta$ moves the economy to the right and results in a reduction in $\gamma_{A B}$. Proposition 9 summarizes the effects on welfare of changing $\eta$ in the type-A equilibrium.

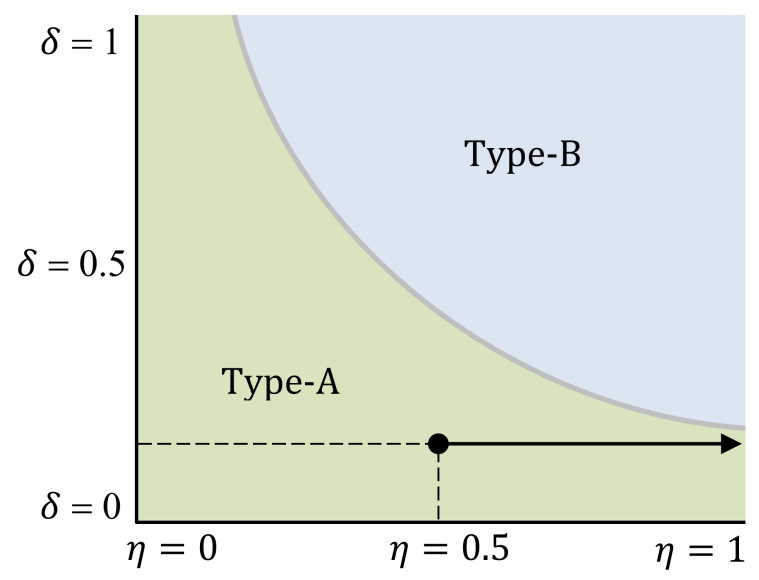

Figure 3: INCREASING $\eta$ IN THE TYPE-A EQUILIBRIUM

Proposition 9 In the type-A equilibrium, it always holds that $\partial \mathcal{W} / \partial \eta<0$.

It is clear that $\partial \hat{q} / \partial \eta=0$, since $u^{\prime}(\hat{q})=1$ for any $\eta$ in the type-A equilibrium. Because $\rho$ only depends on $\beta$, and $g$, we also have $\partial \rho / \partial \eta=0$. Therefore, it also holds that $\partial \ell b / \partial \eta=0$. Proposition 9 states that welfare is decreasing in $\eta$ since $\partial q / \partial \eta<0$. As before, increasing $\eta$ reduces the demand for money, and thus passive agents will consume less in the goods market in the type-A equilibrium. Active agents are not affected by a change in $\eta$ in the type-A equilibrium, since they are able to consume the efficient quantity $u^{\prime}(\hat{q})=1$.

With the knowledge that competitive pricing with probabilistic entry is welfare-decreasing compared with an over-the-counter market which features bargaining, we next analyze what happens if we change $\delta$ at $\eta=1$. We assume that any change in $\delta$ is caused by a change in the matching efficiency $\xi$; i.e., that the fraction of consumers, $1-n$, and producers, $n$, remains unchanged. Increasing $\delta$ moves the economy further in the type-B equilibrium and results in a frictionless centralized market for $\delta=1$, while reducing $\delta$ moves the economy in the type-A equilibrium and results in a complete shutdown of the financial market for $\delta=0$. Note, that $\delta=\xi \mathcal{M}(n, 1-n)(1-n)^{-1}=1$ is only feasible for $n \geq 0.5$, which we assume hereafter. In Figure 4 , we visualize a reduction and an increase in $\delta$ by the vertical arrows for an economy which is initially characterized by $\delta=0.5$ and $\eta=1$, with $C<\bar{C}_{\delta \eta=1}$ and $\gamma>\gamma_{A B}$. 


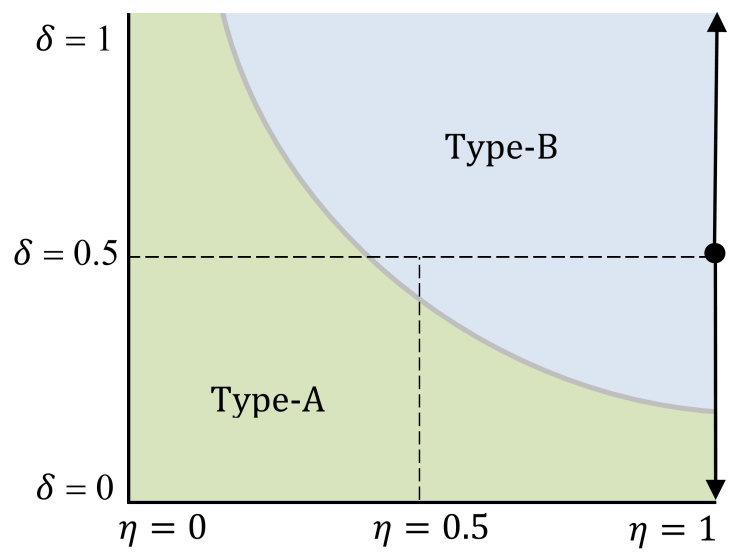

Figure 4: INCREASING OR DECREASING $\delta$

We first analyze the case of increasing $\delta$, which moves the economy upwards in Figure 4; i.e., it reduces $\gamma_{A B, C=\bar{C}}$, since $\partial \gamma_{A B, C=\bar{C}} / \partial \delta<0$. The effects of an increase in $\delta$ are summarized in Proposition 10.

Proposition 10 Let $\delta$ and $\eta$ be large, and $\gamma$ be small in the type- $B$ equilibrium. Then, we have

$$
\frac{\partial \rho}{\partial \delta}>0, \quad \frac{\partial(\ell \mathbb{b})}{\partial \delta}>0, \quad \frac{\partial \hat{q}}{\partial \delta}<0, \quad \frac{\partial q}{\partial \delta}<0, \quad \frac{\partial \mathcal{W}}{\partial \delta}>0 .
$$

Agents take into account that due to the increase in $\delta$ they have a higher trading probability in the financial market, which is why their demand for bonds increases in the centralized market. This effect dominates the larger supply issued by firms, who want to benefit from lower funding costs, such that $\rho$ increases. Hence, there is an increase in trading volume in the financial market which is caused by two effects: (i) more agents enter the market; (ii) more bonds are traded in each match. The drawback of an increase in $\delta$ is that the demand for money of each agent decreases, which reduces the value of money such that active and passive agents consume less in the goods market. Whether an increase in $\delta$ is welfare-improving depends on which of these effects dominates. For low inflation rates, increasing $\delta$ is welfare-improving, because the positive distributional effect of having more trades in the financial market dominates the negative effect on the demand for money.

However, for high inflation rates and large values of $\delta$ and $\eta$, the opposite may occur, which is shown by the following corollary under the additional assumption of $u(q)=\ln q$.

Corollary 11 Let $\delta$ and $\eta$ be large in the type-B equilibrium. Then for large $\gamma$, we have $\partial \mathcal{W} / \partial \delta<$ 0 if $u(q)=\ln q$.

The above corollary confirms the finding of Berentsen et al. (2014), who show that for high inflation rates it can be welfare-improving to increase search frictions in a competitive financial 
market $(\eta=1)$. This is because the effect on the demand for money dominates the distributional effect for high inflation rates and large values of $\delta$ and $\eta$. To the contrary, for high inflation rates and small values of $\delta$ and $\eta$, we cannot derive any analytical results. Therefore, we analyzed our model numerically and found $\partial \mathcal{W} / \partial \delta>0$ holds for high inflation rates and low values of $\delta$ and $\eta$.

Summing up, a frictionless centralized financial market $(\delta \eta=1)$ might indeed improve welfare as compared to an over-the-counter market, if the welfare-improving effect of increasing $\delta$ dominates the adverse effect of increasing $\eta$. However, the more important question is whether a centralized market is superior to having no financial market at all. We therefore analyze in the next paragraph what happens if we reduce $\delta$ in a competitive financial market $(\eta=1)$ with probabilistic entry, which is shown by the downward pointing arrow in Figure 4 . Decreasing $\delta$ moves the economy in the type-A equilibrium, and Proposition 12 summarizes the effects on welfare of decreasing $\delta$ in the type-A equilibrium.

Proposition 12 In the type- $A$ equilibrium, we have $\partial \mathcal{W} / \partial \delta>0$ for small values of $\eta$, and $\partial \mathcal{W} / \partial \delta<0$ for large values of $\eta$.

Following the same reasoning as for the analysis with respect to $\eta$, we have $\partial \hat{q} / \partial \delta=0$, $\partial \rho / \partial \delta=0$, and $\partial \ell \mathrm{b} / \partial \delta=0$ in the type-A equilibrium. Proposition 12 states that for a competitive financial market, welfare is increased by reducing the market access probability $\delta$. The reason is that for high values of $\eta$, the demand for money is too low in the type-A equilibrium. Thus, reducing $\delta$ makes bonds less attractive and induces agents to increase their demand for money, which is welfare-improving. For ease of understanding, we show in Figure 5 the development of welfare as a function of the market access probability $\delta$ for $\eta=1$. As shown in Figure 5 , we find

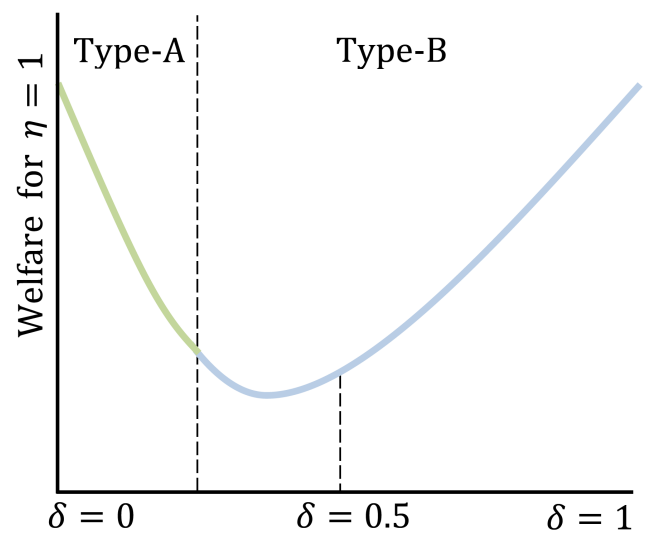

Figure 5: Welfare as FUnCtion of $\delta$ FOR $\eta=1$

that for $\eta=1$, welfare at $\delta=1$ coincides with welfare at $\delta=0$; i.e., $\mathcal{W}_{\delta=1}=\mathcal{W}_{\delta=0}$, while for $\eta<1$ we obtain $\mathcal{W}_{\delta=1}>\mathcal{W}_{\delta=0}$. This finding is formalized in the following lemma. 
Lemma 13 Given $\eta$, let $\delta=0$ support the type- $A$ equilibrium, and $\delta=1$ support the type- $B$ equilibrium. If $\eta=1$, then $\delta=0$ and $\delta=1$ result in an identical value of welfare. Otherwise, $\delta=1$ generates a higher value of welfare than $\delta=0$.

Lemma 13 states that a centralized financial market $(\delta \eta=1)$ is redundant. The reason is the following. When the competitive financial market is shut down $(\delta=0$ and $\eta=1)$, we are in the type-A equilibrium. Since all agents are passive, the demand for bonds is low and thus they are priced at their fundamental value, $\rho=\beta(1-g)$, while the quantity consumed by passive agents, equation (14), satisfies $\gamma / \beta=(1-n) u^{\prime}(q)+n$. To the contrary, when the financial market is centralized $(\delta \eta=1)$, agents are certain to trade in the financial market. Consequently, their demand for bonds is so high that the issuance price of bonds increases to $\rho=\gamma(1-g)$; i.e., the return on bonds decreases until equaling the return on money. Additionally, the demand for money decreases rapidly, such that the quantity consumed by active agents, equation (17), satisfies $\gamma / \beta=(1-n) u^{\prime}(\hat{q})+n$; i.e., after trading in the financial market, agents are only able to consume the same quantity that would be attainable if no financial market existed at all.

\section{Optimal Policy}

We now analyze the optimal policy in a financial market which initially features search and bargaining. As before, we analyze an economy which is initially characterized by $\delta=\eta=0.5$ with $\gamma>\gamma_{A B}$ and $C<\bar{C}_{\delta \eta=1}$. In Figure 6, we show the optimal intervention in such an environment using the black arrow. Furthermore, we highlight in yellow the area in terms of search frictions $(\delta)$ and the bargaining power of consumers $(\eta)$, which is superior to having a centralized market; i.e., which features higher welfare than there would be if no financial market existed.

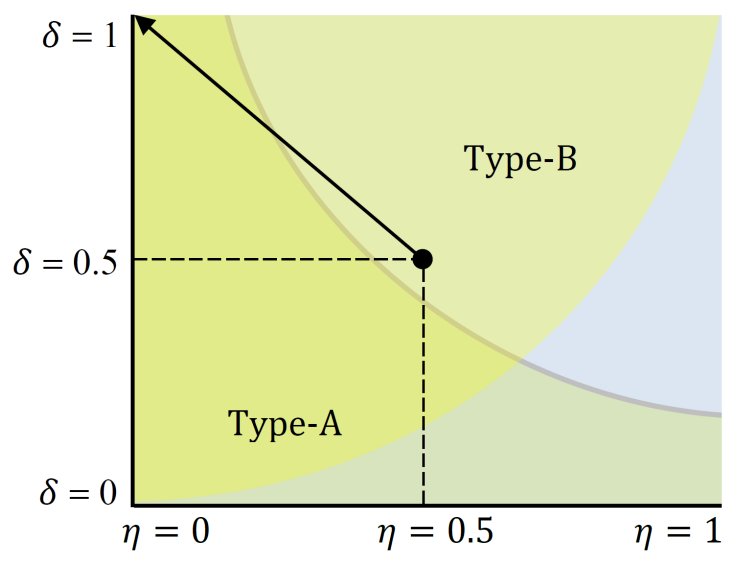

Figure 6: Optimal POLICY

Figure 6 shows that the optimal intervention moves the economy to the top-left corner in the 
type-A equilibrium. From Proposition 7 and 10, we already know that $\partial \mathcal{W} / \partial \eta<0$ and $\partial \mathcal{W} / \partial \delta>$ 0 in the type-B equilibrium for large values of $\delta$ and $\eta$ and low inflation rates. Furthermore, from Proposition 9 and 12, we know that in the type-A equilibrium we have $\partial \mathcal{W} / \partial \eta<0$ and $\partial \mathcal{W} / \partial \delta>0$ for small values of $\eta$. Hence, reducing $\eta$ and increasing $\delta$ both have welfare-enhancing effects in the type-A equilibrium. Reducing $\eta$ improves welfare, because it incentivizes agents to increase their demand for money, which in turn allows them to consume more in the goods market. For small values of $\eta$, increasing $\delta$ is welfare-improving, because the distributional effect of having a better allocation of the medium of exchange dominates the negative effect on the demand for money. For low inflation rates, the combination of both effects $(\delta=1$ and $\eta=0)$ results in the optimal intervention in a financial market which initially features search frictions $(0<\delta<1)$ and bargaining $(0<\eta<1)$.

However, for higher inflation rates the type-A equilibrium ceases to exist and the economy remains in the type-B equilibrium for any value of $\delta$ and $\eta$. Although we cannot prove analytically that Proposition 7 and 10 also hold for high inflation rates and small values of $\delta$ and $\eta$, we found numerically that reducing $\eta$ and increasing $\delta$ remains the optimal policy for high inflation rates in the type-B equilibrium.

The yellow area in Figure 6 highlights that for a wide range of parameter values, over-thecounter trading achieves a superior allocation in comparison to a centralized market. We formalize this finding in the following lemma.

Lemma 14 For any given $\gamma, \beta, \eta, n, C$, and $g$, if

$$
\frac{u(x)-x-[u(q)-q]}{u(\hat{q})-\hat{q}-[u(q)-q]}<\delta
$$

where (i) $\hat{q}, q$ satisfies either equations (13), (14), (15) or equations (16), (17), (18), and (ii) $x$ satisfies

$$
\frac{\gamma}{\beta}=(1-n) u^{\prime}(x)+n,
$$

then over-the-counter trading is superior to having no financial market at all.

Note that if $\eta<1$, then the left-hand side of $(21)$ is smaller than 1 for any $0<\delta \leq 1$. Lemma 14 states that if $\eta<1$ and $\delta$ is large enough, then it is always better to have an over-the-counter market as opposed to having no financial market at all. Moreover, if $\eta=0$, then $q=x$. Hence, for any $0<\delta \leq 1$, the left-hand side of (21) equals zero. Therefore, if $\eta=0$, then it is beneficial to have an over-the-counter market for any $0<\delta \leq 1$. In addition, if $\eta=1$, then the inequality (21) is not satisfied for any $0<\delta \leq 1$ by Lemma 13. That is, having a competitive market with probabilistic entry $(0<\delta \leq 1)$ is inferior to having an over-the-counter market that features bargaining $(\eta<1)$. 


\section{Discussion}

In this section, we discuss further equilibria, the relation of our work to previous studies, and the differentiation between government bonds and corporate bonds.

Further equilibria and the relation to previous studies. So far, we have analyzed an equilibrium where both money and bonds are plentiful (type-A), and an equilibrium where bonds are scarce and money is plentiful (type-B). However, there exists at least a third equilibrium, where money is scarce and bonds are plentiful, denoted as type-C. In this equilibrium, a matched consumer will consume less than $q^{*}$ because the producer runs out of money before the consumer sells all of his bonds. As a consequence, bonds are priced at their fundamental value, $\rho=\beta(1-g)$. The type-C equilibrium exists if, and only if, $\gamma>\gamma_{A B, C=\bar{C}}$ and $C>\bar{C}$. We have not reported this equilibrium in the main text of the paper for two reasons: First, competitive pricing and over-the-counter trading do not coincide for $\eta=1$. Second, the regions of existence become more cumbersome, because there exists a fourth equilibrium under competitive pricing for $\gamma>\gamma_{A B, C=\bar{C}}$ and $C>\bar{C}$, denoted as type-D, where both money and bonds are scarce. Figure 7 shows the regions of existence for the two market structures, with over-the-counter trading shown on the left-hand side and competitive pricing with probabilistic entry shown on the right-hand side.

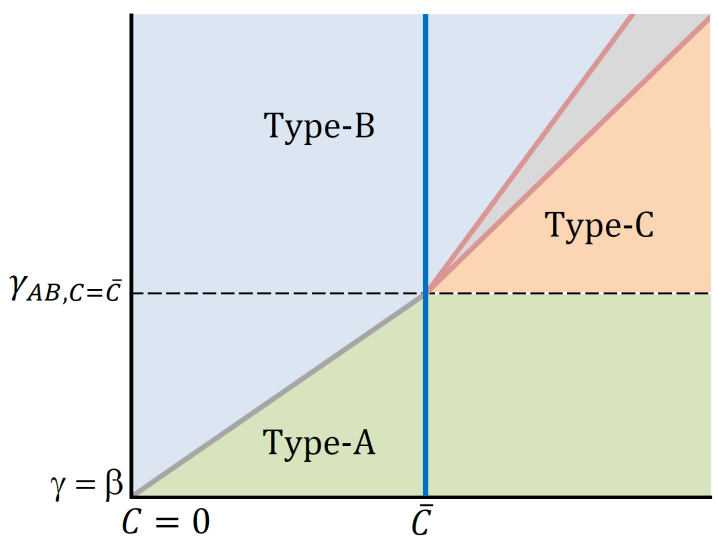

Over-the-Counter: $\eta<1$ and $\delta<1$

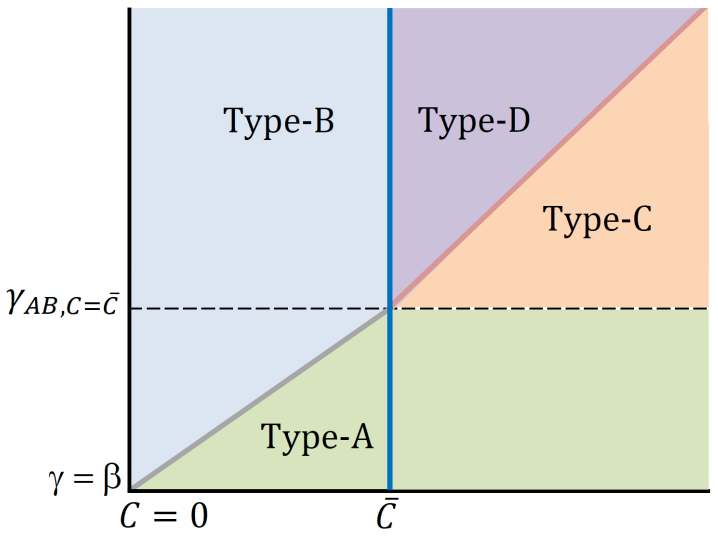

Competitive Pricing: $\delta<1$

\section{Figure 7: Further Equilibria}

For an over-the-counter financial market with $0<\delta \eta<1$, the type-C equilibrium exists for $\gamma>\gamma_{A B, C=\bar{C}}$ and $C>\bar{C}$ (see the left-hand side diagram of Figure 7 ). The gray area on the top-right corner marks a region where either the type-B and the type- $\mathrm{C}$ equilibrium coexist or none of the above mentioned equilibria exist. Which case prevails depends on parameter values. In the latter case, financial intermediation shuts down, and the allocation is characterized by the standard Lagos and Wright (2005) model. 
For a competitive financial market with probabilistic entry, the type-C equilibrium also exists for $\gamma>\gamma_{A B, C=\bar{C}}$ and $C>\bar{C}$ (see the right-hand side diagram of Figure 7). However, the equilibrium equations for Kalai bargaining and competitive pricing do not coincide for $\eta=1$. Furthermore, if inflation is high enough, the type-D equilibrium exists, where both money and bonds are scarce and bonds are priced above their fundamental value.

Due to the these irregularities, we only treat the type-A and the type-B equilibrium in the main text. However, having a look at the entire picture allows us to differentiate our work in more detail from previous studies. For instance, Berentsen et al. (2014) show that for a competitive financial market, welfare is improved by reducing $\delta$ in the type-D equilibrium. In Corollary 11, we confirm that the findings of Berentsen et al. (2014) also hold in the type-B equilibrium for high inflation rates and large values of $\delta$ and $\eta \cdot{ }^{15}$ However, our study goes a step further and shows that for a wide range of parameter values, an over-the-counter market achieves a superior allocation when compared with a competitive financial market with probabilistic access. Furthermore, we show that for any inflation rate above the Friedman rule, the optimal policy is to increase $\delta$ and to reduce $\eta$. This intervention reduces consumption variability and achieves a superior allocation when compared with the optimal policy proposed in Berentsen et al. (2014), which increases consumption variability.

Geromichalos and Herrenbrueck (2016a) show that welfare in an over-the-counter market, i.e., "search and bargaining" taken together, can be higher than in a competitive market for $\delta=1$. Under this condition, the type-A equilibrium ceases to exist under competitive pricing $(\eta=1$ and $\gamma_{A B, C=\bar{C}}=\beta$ ) for any $\gamma>\beta$, while it continues to exist under Kalai bargaining for $\eta<1$ and low inflation rates. That is, for low enough inflation rates, all consumers are able to consume $q^{*}$ under Kalai bargaining (since $\delta=1$ ), while they consume $q<q^{*}$ under competitive pricing in the type-B, type-C, and type-D equilibrium. In contrast to Geromichalos and Herrenbrueck (2016a), we disentangle the effects of search and bargaining on welfare. Concretely, we show that the role of search frictions is quite different from the one of bargaining. Search frictions decrease the demand for money, but also improve the allocation of the medium of exchange. We show that the latter effect dominates the former for a wide range of parameter values. On the other hand, bargaining increases the demand for money and is therefore welfare improving for any $\gamma>\beta$. Finally, we show that for any inflation rate above the Friedman rule the optimal policy is to increase $\delta$ and to reduce $\eta$. These are aspects regarding the optimality of over-the-counter markets which are not analyzed by Geromichalos and Herrenbrueck (2016a).

Government bonds vs. corporate bonds. Throughout the paper, the supply of bonds has been endogenous and bonds were issued in real terms by generic firms. We showed that in such an environment, it is typically inadvisable to move corporate bond markets from an over-the-counter structure to a centralized market. However, we were silent about another major bond market, namely the market for government bonds. In order to analyze whether our results continue to hold for this market, we have also derived our model for nominal government bonds. To do this,

\footnotetext{
${ }^{15}$ Since welfare is continuous in each type of equilibrium and since welfare is maximized for $0<\delta<1$ in the type-D equilibrium, then it must also be maximized for $0<\delta<1$ in the type-B equilibrium for large values of $\eta$ by above continuities.
} 
we have assumed that these assets are issued by the central bank, which alters the government budget constraint from $\tau M_{-1}=M-M_{-1}$ to

$$
M-M_{-1}=\tau M_{-1}+B_{-1}-\rho B
$$

where $B_{-1}$ denotes the money creation to redeem previously issued bonds at $\rho=1$, and $\rho B$ the reduction in the money supply from selling $B$ units of bonds at price $\rho \leq 1$. Since the central bank can print any amount of money to redeem bonds, we assume that $g=0$ for government bonds. In equilibrium, the gross growth rate of the supply of bonds equals the gross growth rate of the supply of money, which is independent of $\eta$ and $\delta$. A further difference between corporate bonds and government bonds is that the former are issued in real terms, while the latter are issued in nominal terms. Therefore, in the type-B equilibrium for $\delta \eta=1$ we obtain a bond price equaling $\rho=\gamma$ for real bonds and $\rho=1$ for nominal bonds when $g=0$. Hence, for both specifications, the return on bonds decreases until equaling the return on money for $\delta \eta=1$ in the type-B equilibrium. Furthermore, we find that the sign of the derivatives presented in Propositions 7, 9, 10, and 12 remains the same (except for the effect on the issued quantity of bonds, which is exogenous for government bonds); i.e., our results also hold for the government bond market.

\section{Conclusion}

In this paper, we present a model that incorporates an over-the-counter financial market and show that it is generally inadvisable to centralize this market. To do this, we disentangle the effects of search and bargaining on welfare. We find that bargaining induces agents to increase their demand for the liquid asset, which increases its value and so consumption of all market participants. Search frictions also induce agents to increase their demand for the liquid asset, however, they also imply a less efficient allocation of the medium of exchange. We show that for a wide range of parameter values the latter effect dominates the former, such that it is optimal to reduce search frictions and to reduce the bargaining power of liquidity demanders. Although it might not be feasible for policy makers to affect the bargaining power of financial market participants, our results imply that having financial intermediaries who charge bid-ask spreads to investors is good from a societal point of view. This is because intermediaries are able to extract part of the trade surplus and thereby provoke the welfare-improving effect postulated by our model.

Furthermore, we endogenize the supply of the illiquid asset. This allows us to show that for high inflation rates and/or low trading frictions, the illiquid asset exhibits a liquidity premium. In a such an environment, firms intend to benefit from the lower funding costs, which is why the total supply of the illiquid asset increases. Our model thus also delivers an explanation about why the supply of financial assets which are traded in markets with high trading frictions is generally lower than the one of financial assets which are traded in markets with low trading frictions. 


\section{A Appendix - For Online Publication}

Proof of Proposition 1. Derivation of (13). It is obvious that (13) holds when the shortselling constraints (6) are non-binding in (8).

Derivation of (14). At the beginning of a representative period, the marginal value of money is given by

$$
\frac{\partial V_{1}}{\partial m}=(1-n) \frac{\partial V_{1}^{c}}{\partial m}+n \frac{\partial V_{1}^{p}}{\partial m} .
$$

By (10), the above equation can be rewritten as

$$
\frac{\partial V_{1}}{\partial m}=(1-n)\left[\delta \eta \frac{\partial K}{\partial m_{c}}+\frac{\partial V_{2}^{c}}{\partial m_{c}}\right]+n\left[\delta_{p}(1-\eta) \frac{\partial K}{\partial m_{p}}+\frac{\partial V_{2}^{p}}{\partial m_{p}}\right]
$$

where $\partial K / \partial m_{j}:=\partial K\left(m_{c}, m_{p}, b_{c}, b_{p}\right) /\left.\partial m_{j}\right|_{\left(m_{c}, m_{p}, b_{c}, b_{p}\right)=(m, m, b, b)}$ for $j=c, p$. Next, we derive the first-order and the envelope conditions in the centralized market and the envelope conditions in the goods market to replace $\partial V_{1} / \partial m, \partial V_{2}^{c} / \partial m_{c}$ and $\partial V_{2}^{p} / \partial m_{p}$, respectively. In the centralized market, the first-order conditions with respect to $m_{+1}, b_{+1}$ and $x$ are $U^{\prime}(x)=1$, and

$$
\frac{\beta \partial V_{1}}{\partial m_{+1}}=\frac{\phi}{\rho} \frac{\beta \partial V_{1}}{\partial b_{+1}}=\phi
$$

It is standard in models building on Lagos and Wright (2005) that the choice of $m_{+1}$ and $b_{+1}$ is independent of $m$ and $b$, which results in a degenerate distribution of money and bonds at the beginning of a period. Using $\beta \partial V_{1} / \partial m_{+1}$ updated one period, we can replace $\partial V_{1} / \partial m$ in $(22)$. The envelope conditions in the centralized market are

$$
\frac{\partial V_{3}}{\partial m}=\frac{\phi}{1-g} \frac{\partial V_{3}}{\partial b}=\phi .
$$

In the goods market, the buyer's envelope conditions are

$$
\frac{\partial V_{2}^{c}}{\partial m}=\phi u^{\prime}(q) \quad \text { and } \quad \frac{\partial V_{2}^{c}}{\partial b}=1-g,
$$

where we used (24) to replace $\partial V_{3} / \partial m$ and $\partial V_{3} / \partial b$. Following the same procedure, we obtain the producer's envelope conditions in the goods market as

$$
\frac{\partial V_{2}^{p}}{\partial m}=\frac{\phi}{1-g} \frac{\partial V_{2}^{p}}{\partial b}=\phi
$$

Next, we need to derive the envelope conditions in the centralized market to replace $\partial K / \partial m_{c}$ and $\partial K / \partial m_{p}$ in $(22)$. We obtain

$$
\frac{\partial K}{\partial m_{c}}=\phi-\phi u^{\prime}(q) \quad \text { and } \quad \frac{\partial K}{\partial m_{p}}=0
$$


where we have used $\partial \hat{q} / \partial m_{c}=0, \partial q / \partial m_{c}=1 / p=\phi, \partial d_{m} / \partial m_{c}=-1$, and $\partial \hat{q} / \partial m_{p}=\partial q / \partial m_{p}=$ $\partial d_{m} / \partial m_{p}=0$ by (9). Using (23), (25), (26), and (27) in (22), we obtain (14).

Derivation of (15). We follow the same procedure as in the derivation of (22) to rewrite the marginal value of bonds at the beginning of a period as

$$
\frac{\partial V_{1}}{\partial b}=(1-n)\left[\delta \eta \frac{\partial K}{\partial b_{c}}+\frac{\partial V_{2}^{c}}{\partial b_{c}}\right]+n\left[\delta_{p}(1-\eta) \frac{\partial K}{\partial b_{p}}+\frac{\partial V_{2}^{p}}{\partial b_{p}}\right] .
$$

To replace $\partial V_{2}^{c} / \partial b_{c}$ and $\partial V_{2}^{p} / \partial b_{p}$, we use (25) and (26), respectively. Because the bond constraint is non-binding in the type-A equilibrium, we have $\partial K / \partial b_{p}=\partial K / \partial b_{c}=0$. Finally, using (23) updated one period, we obtain (15).

Proof of Proposition 2. Derivation of (16). As a consequence of the binding bond constraint of active consumers, it holds that $d_{b}=b$. Furthermore, since the cash constraint of consumers (active and passive) is binding in the goods market, it holds that $m_{c}+d_{m}=p \hat{q}$ and $m_{c}=p q$. Using these expressions in (7) and the first-order condition of producers in the goods market to replace $p \phi=1$, we obtain (16).

Derivation of (17). At the beginning of a representative period, the marginal value of money is given by

$$
\frac{\partial V_{1}}{\partial m}=(1-n)\left[\delta \eta \frac{\partial K}{\partial m_{c}}+\frac{\partial V_{2}^{c}}{\partial m_{c}}\right]+n\left[\delta_{p}(1-\eta) \frac{\partial K}{\partial m_{p}}+\frac{\partial V_{2}^{p}}{\partial m_{p}}\right],
$$

where we used (10) to replace $V_{1}^{c}\left(m_{c}, b_{c}\right)$ and $V_{1}^{p}\left(m_{c}, b_{c}\right)$. We use the envelope conditions of consumers and producers in the goods market, (25) and (26), to replace $\partial V_{2}^{c} / \partial m_{c}$ and $\partial V_{2}^{p} / \partial m_{p}$, respectively. Furthermore, because the producer's cash constraint is non-binding, we can use $\partial \hat{q} / \partial m_{p}=\partial q / \partial m_{p}=\partial d_{m} / \partial m_{p}=0$ with (9) to obtain $\partial K / \partial m_{p}=0$. Using these expressions to rewrite the marginal value of money at the beginning of a period, we obtain

$$
\frac{\partial V_{1}}{\partial m}=(1-n)\left[\delta \eta \frac{\partial K}{\partial m_{c}}+\phi u^{\prime}(q)\right]+n \phi
$$

The envelope condition of a consumer in the financial market, $\partial K / \partial m_{c}$, is derived as follows. Using (9), we obtain

$$
\frac{\partial K}{\partial m_{c}}=u^{\prime}(\hat{q}) \frac{\partial \hat{q}}{\partial m_{c}}-u^{\prime}(q) \frac{\partial q}{\partial m_{c}}-\phi \frac{\partial d_{m}}{\partial m_{c}}
$$

Next, we need to derive $\partial \hat{q} / \partial m_{c}, \partial q / \partial m_{c}$ and $\partial d_{m} / \partial m_{c}$. Using (7), we obtain

$$
(1-\eta)\left[u^{\prime}(\hat{q}) \frac{\partial \hat{q}}{\partial m_{c}}-u^{\prime}(q) \frac{\partial q}{\partial m_{c}}\right]=-\eta \phi \frac{\partial d_{m}}{\partial m_{c}}
$$

and because $\hat{q}=\phi\left(m_{c}+d_{m}\right), q=\phi m_{c}$, we also obtain

$$
\frac{\partial \hat{q}}{\partial m_{c}}=\phi+\phi \frac{\partial d_{m}}{\partial m_{c}} \quad \text { and } \quad \frac{\partial q}{\partial m_{c}}=\phi .
$$


Using the above expressions for $\partial \hat{q} / \partial m_{c}$ and $\partial q / \partial m_{c}$ in (30), we obtain

$$
\begin{aligned}
\frac{\partial \hat{q}}{\partial m_{c}} & =\phi \frac{\eta+(1-\eta) u^{\prime}(q)}{\eta+(1-\eta) u^{\prime}(\hat{q})} \\
\frac{\partial d_{m}}{\partial m_{c}} & =-\frac{1-\eta}{\phi \eta}\left[u^{\prime}(\hat{q}) \frac{\partial \hat{q}}{\partial m_{c}}-u^{\prime}(q) \frac{\partial q}{\partial m_{c}}\right] .
\end{aligned}
$$

Using the above expressions for $\partial \hat{q} / \partial m_{c}, \partial d_{m} / \partial m_{c}$, and $\partial q / \partial m_{c}=\phi$ in (29), we obtain the following expression for $\partial K / \partial m_{c}$ :

$$
\begin{aligned}
\frac{\partial K}{\partial m_{c}} & =u^{\prime}(\hat{q}) \frac{\partial \hat{q}}{\partial m_{c}}-u^{\prime}(q) \frac{\partial q}{\partial m_{c}}+\frac{1-\eta}{\eta}\left[u^{\prime}(\hat{q}) \frac{\partial \hat{q}}{\partial m_{c}}-u^{\prime}(q) \frac{\partial q}{\partial m_{c}}\right] \\
& =\frac{1}{\eta}\left[u^{\prime}(\hat{q}) \frac{\partial \hat{q}}{\partial m_{c}}-u^{\prime}(q) \frac{\partial q}{\partial m_{c}}\right]=\frac{\phi}{\eta}\left[u^{\prime}(\hat{q}) \frac{\eta+(1-\eta) u^{\prime}(q)}{\eta+(1-\eta) u^{\prime}(\hat{q})}-u^{\prime}(q)\right]
\end{aligned}
$$

Using the above solution to replace $\partial K / \partial m_{c}$ in (28) and (23) updated one period to replace $\partial V_{1} / \partial m$, we obtain (17).

Derivation of (18). At the beginning of a representative period, the marginal value of bonds is given by

$$
\frac{\partial V_{1}}{\partial b}=(1-n)\left[\delta \eta \frac{\partial K}{\partial b_{c}}+\frac{\partial V_{2}^{c}}{\partial b_{c}}\right]+n\left[\delta_{p}(1-\eta) \frac{\partial K}{\partial b_{p}}+\frac{\partial V_{2}^{p}}{\partial b_{p}}\right] .
$$

Using (16), we can obtain

$$
\frac{\partial \hat{q}}{\partial b}=\frac{(1-g)}{(1-\eta) u^{\prime}(\hat{q})+\eta} \quad \text { and } \quad \frac{\partial d_{m}}{\partial b}=\frac{1}{\phi} \frac{1-g}{(1-\eta) u^{\prime}(\hat{q})+\eta},
$$

where we used $\ell \mathbb{b}=b$ and $\phi d_{m}=\hat{q}-q$. We can use the above expressions to replace $\partial \hat{q} / \partial b$ and $\partial d_{m} / \partial b$, respectively, $\partial q / \partial b_{c}=0$, and $\partial \hat{q} / \partial b_{p}=\partial q / \partial b_{p}=\partial d_{m} / \partial b_{p}=0$, in (9), to obtain

$$
\begin{aligned}
& \frac{\partial K}{\partial b_{c}}=(1-g) \frac{u^{\prime}(\hat{q})-1}{(1-\eta) u^{\prime}(\hat{q})+\eta} \\
& \frac{\partial K}{\partial b_{p}}=0
\end{aligned}
$$

Using the two above expressions, we can replace $\partial K / \partial b_{c}$ and $\partial K / \partial b_{p}$ in (34); using the envelope conditions in the goods market, (25) and (26), we can replace $\partial V_{2}^{c} / \partial b_{c}$ and $\partial V_{2}^{p} / \partial b_{p}$; and using (23) updated one period we can replace $\partial V_{1} / \partial b$ to obtain (18).

Proof of Lemma 3. By (11) and (12), we obtain

$$
\frac{1-\beta(1-g)}{(1-g)} C-\frac{f(\rho \mathfrak{b})}{\rho f^{\prime}(\rho \mathfrak{b})}+\mathfrak{b}=0 .
$$


Hence,

$$
\begin{aligned}
& \frac{\partial \mathfrak{b}}{\partial C}=-\frac{1-\beta(1-g)}{(1-g)} \frac{\left[f^{\prime}(\rho \mathfrak{b})\right]^{2}}{f(\rho \mathfrak{b}) f^{\prime \prime}(\rho \mathfrak{b})}=\frac{1-\beta(1-g)}{(1-g)} \frac{\epsilon(\rho \mathfrak{b})}{\alpha(\rho \mathfrak{b})}>0, \\
& \frac{\partial \mathfrak{b}}{\partial g}=-\frac{C}{(1-g)^{2}} \frac{\left[f^{\prime}(\rho \mathfrak{b})\right]^{2}}{f(\rho \mathfrak{b}) f^{\prime \prime}(\rho \mathfrak{b})}=\frac{C}{(1-g)^{2}} \frac{\epsilon(\rho \mathfrak{b})}{\alpha(\rho \mathfrak{b})}>0, \\
& \frac{\partial \mathfrak{b}}{\partial \beta}=C \frac{\left[f^{\prime}(\rho \mathfrak{b})\right]^{2}}{f(\rho \mathfrak{b}) f^{\prime \prime}(\rho \mathfrak{b})}=-C \frac{\epsilon(\rho \mathfrak{b})}{\alpha(\rho \mathrm{b})}<0 .
\end{aligned}
$$

Using (11), we obtain

$$
\frac{\partial \ell}{\partial \mathfrak{b}}=\rho^{2} f^{\prime \prime}(\rho \mathbb{b})<0 .
$$

Thus, we obtain

$$
\frac{\partial \ell}{\partial C}<0, \quad \frac{\partial \ell}{\partial g}<0, \quad \frac{\partial \ell}{\partial \beta}>0 .
$$

Using (11), we also obtain

$$
\ell \mathfrak{b}=f^{\prime}(\rho \mathfrak{b}) \rho \mathfrak{b}
$$

Consequently, we obtain

$$
\frac{\partial \ell \mathfrak{b}}{\partial \mathfrak{b}}=\rho f^{\prime}(\rho \mathfrak{b})\left[1+\frac{\rho \mathfrak{b} f^{\prime \prime}(\rho \mathfrak{b})}{f^{\prime}(\rho \mathfrak{b})}\right]=\rho f^{\prime}(\rho \mathfrak{b})[1-\alpha(\rho \mathfrak{b})] .
$$

By using

$$
\frac{\partial \ell \mathfrak{b}}{\partial C}=\frac{\partial \ell \mathbb{b}}{\partial \mathfrak{b}} \frac{\partial \mathfrak{b}}{\partial C}=\rho f^{\prime}(\rho \mathbb{b})[1-\alpha(\rho \mathbb{b})] \frac{\partial \mathfrak{b}}{\partial C},
$$

we obtain the desired result for $\partial \ell \mathrm{b} / \partial C$. It is clear that when we follow the above procedure, we can derive the results for $\partial \ell \mathrm{b} / \partial g$, and $\partial \ell \mathrm{b} / \partial \beta$.

Proof of Lemma 4. By (11) and (12), we obtain

$$
\frac{1-\beta(1-g)}{(1-g)} C-\frac{f(\rho \mathfrak{b})}{\rho f^{\prime}(\rho \mathfrak{b})}+\mathfrak{b}=0 .
$$

Therefore, we obtain

$$
\begin{aligned}
& f(\rho \mathbb{b}) f^{\prime \prime}(\rho \mathbb{b}) \rho^{2} \frac{\partial \mathfrak{b}}{\partial \rho}=\left[f^{\prime}(\rho \mathfrak{b})\right]^{2} \rho \mathfrak{b}-f(\rho \mathbb{b}) f^{\prime}(\rho \mathbb{b})-f(\rho \mathfrak{b}) f^{\prime \prime}(\rho \mathbb{b}) \rho \mathbb{b} \\
& =f(\rho \mathrm{b}) f^{\prime}(\rho \mathrm{b})\left[\frac{f^{\prime}(\rho \mathrm{b}) \rho \mathrm{b}}{f(\rho \mathrm{b})}-1-\frac{f^{\prime \prime}(\rho \mathrm{b}) \rho \mathrm{b}}{f^{\prime}(\rho \mathrm{b})}\right] \\
& =-f(\rho \mathfrak{b}) f^{\prime}(\rho \mathfrak{b})[1-\epsilon(\rho \mathfrak{b})-\alpha(\rho \mathfrak{b})] \text {. }
\end{aligned}
$$


Rearranging terms yields

$$
\frac{\partial \mathfrak{b}}{\partial \rho}=\frac{\mathfrak{b}}{\alpha(\rho \mathbb{b}) \rho}[1-\epsilon(\rho \mathfrak{b})-\alpha(\rho \mathbb{b})] .
$$

From $\ell \mathfrak{b}=f^{\prime}(\rho \mathfrak{b}) \rho \mathfrak{b}$, we obtain

$$
\frac{\partial \ell \mathfrak{b}}{\partial \rho}=f^{\prime \prime}(\rho \mathfrak{b}) \rho \mathfrak{b}^{2}+f^{\prime \prime}(\rho \mathfrak{b}) \rho^{2} \mathfrak{b} \frac{\partial \mathfrak{b}}{\partial \rho}+f^{\prime}(\rho \mathfrak{b}) \mathfrak{b}+f^{\prime}(\rho \mathfrak{b}) \rho \frac{\partial \mathfrak{b}}{\partial \rho} .
$$

Using the above equation and (35), we obtain

$$
\begin{aligned}
& \frac{\partial \ell \mathfrak{b}}{\partial \rho}=\frac{\left[f^{\prime}(\rho \mathfrak{b})\right]^{2} \rho \mathfrak{b}^{2}}{f(\rho \mathfrak{b})}+f^{\prime}(\rho \mathfrak{b}) \rho \frac{\partial \mathfrak{b}}{\partial \rho} \\
& =\frac{f^{\prime}(\rho \mathfrak{b})}{f(\rho \mathfrak{b}) f^{\prime \prime}(\rho \mathfrak{b}) \rho}\left[f^{\prime}(\rho \mathfrak{b}) f^{\prime \prime}(\rho \mathfrak{b}) \rho^{2} \mathfrak{b}^{2}+f(\rho \mathfrak{b}) f^{\prime \prime}(\rho \mathfrak{b}) \rho^{2} \frac{\partial \mathfrak{b}}{\partial \rho}\right] \\
& =\frac{f^{\prime}(\rho \mathfrak{b})}{f(\rho \mathfrak{b}) f^{\prime \prime}(\rho \mathfrak{b}) \rho}\left[f^{\prime}(\rho \mathfrak{b}) f^{\prime \prime}(\rho \mathfrak{b}) \rho^{2} \mathfrak{b}^{2}+\left[f^{\prime}(\rho \mathfrak{b})\right]^{2} \rho \mathfrak{b}-f(\rho \mathfrak{b}) f^{\prime}(\rho \mathfrak{b})-f(\rho \mathfrak{b}) f^{\prime \prime}(\rho \mathfrak{b}) \rho \mathfrak{b}\right] \\
& =\frac{\left[f^{\prime}(\rho \mathbb{b})\right]^{2}}{f(\rho \mathfrak{b}) f^{\prime \prime}(\rho \mathbb{b}) \rho}\left[f^{\prime}(\rho \mathfrak{b}) \rho \mathbb{b}-f(\rho \mathfrak{b})\right]\left[1+\frac{f^{\prime \prime}(\rho \mathfrak{b}) \rho \mathfrak{b}}{f^{\prime}(\rho \mathfrak{b})}\right] \\
& =-\frac{\left[f^{\prime}(\rho \mathfrak{b})\right]^{2}}{f^{\prime \prime}(\rho \mathfrak{b}) \rho}[1-\epsilon(\rho \mathfrak{b})][1-\alpha(\rho \mathfrak{b})] \text {. }
\end{aligned}
$$

Because $f(x)$ is concave, it follows that $1-\epsilon(\rho \mathrm{b})>0$ and thus that

$$
\frac{\partial \ell \mathfrak{b}}{\partial \rho} \begin{cases}<0, & \text { if } \alpha(\rho \mathfrak{b})>1 \\ =0, & \text { if } \alpha(\rho \mathfrak{b})=1 \\ >0, & \text { if } \alpha(\rho \mathfrak{b})<1\end{cases}
$$

Finally, note that

$$
\frac{\partial \ell \mathfrak{b}}{\partial \rho}=\frac{\partial \ell}{\partial \rho} \mathbb{b}+\ell \frac{\partial \mathfrak{b}}{\partial \rho}=\frac{\partial \ell}{\partial \rho} \mathfrak{b}+f^{\prime}(\rho \mathfrak{b}) \rho \frac{\partial \mathfrak{b}}{\partial \rho} .
$$

Using (36), we obtain

$$
\frac{\partial \ell}{\partial \rho}=\frac{\left[f^{\prime}(\rho \mathbb{b})\right]^{2} \rho \mathbb{b}}{f(\rho \mathbb{b})}=\epsilon(\rho \mathbb{b}) f^{\prime}(\rho \mathbb{b})
$$

Proof of Proposition 5. First, we find the critical value $\bar{C}$ which is the value of $C$ where the cash constraint of active producers becomes just binding; i.e., $m_{p}=d_{m}=M$. Furthermore, since active and passive consumers are cash-constrained in the goods market, we have $m_{c}+d_{m}=p \hat{q}$ and $m_{c}=p q$, respectively. Using $m_{p}=m_{c}=M$ and rearranging terms, we obtain $\hat{q}=2 q$. 
Furthermore, we have $u^{\prime}(\hat{q})=1$ and

$$
\begin{aligned}
\gamma_{2} & =\beta\left\{(1-n)\left[\delta \eta+(1-\delta \eta) u^{\prime}\left(q^{*} / 2\right)\right]+n\right\}, \\
\rho_{2} & =\beta(1-g), \\
\ell_{2} & =\rho_{2} f^{\prime}\left(\rho_{2} \mathfrak{b}_{2}\right), \\
\mathfrak{b}_{2} & =\frac{(1-\eta)\left[u\left(q^{*}\right)-u\left(q^{*} / 2\right)\right]+\eta q^{*} / 2}{(1-g) \ell_{2}},
\end{aligned}
$$

The critical entry cost $\bar{C}$ is the solution to

$$
\bar{C} \equiv \frac{(1-g)}{1-\beta(1-g)}\left[\frac{f\left(\rho_{2} \mathfrak{b}_{2}\right)}{\ell_{2}}-\mathfrak{b}_{2}\right] .
$$

The critical inflation rate $\gamma_{A B}$ is the value of $\gamma$, where the bond constraint of active consumers becomes just binding in the type-A equilibrium; i.e., (16) holds. For a given $C<\bar{C}$, let $q_{1}, \rho_{1}$, $\ell_{1}$, and $\mathbb{b}_{1}$ satisfy

$$
\begin{aligned}
\frac{\gamma}{\beta} & =(1-n)\left[\delta \eta+(1-\delta \eta) u^{\prime}\left(q_{1}\right)\right]+n, \\
\rho_{1} & =\beta(1-g), \\
\ell_{1} & =\rho_{1} f^{\prime}\left(\rho_{1} \mathfrak{b}_{1}\right), \\
\mathfrak{b}_{1} & =\frac{(1-\eta)\left[u\left(q^{*}\right)-u\left(q_{1}\right)\right]+\eta\left(q^{*}-q_{1}\right)}{(1-g) \ell_{1}},
\end{aligned}
$$

where we used $u^{\prime}(\hat{q})=1$. The critical inflation rate $\gamma_{A B}$ is the solution to

$$
C \equiv \frac{(1-g)}{1-\beta(1-g)}\left[\frac{f\left(\rho_{1} \mathfrak{b}_{1}\right)}{\ell_{1}}-\mathfrak{b}_{1}\right] \text {. }
$$

Proof of Proposition 6. We now assume competitive pricing in the financial market, which changes the agent's decision problem in the following way. As before, an agent enters the financial market with a portfolio $\left(m_{j}, b_{j}\right)$ and leaves it with a portfolio $\left(\hat{m}_{j}, \hat{b}_{j}\right)$, where $j=c$ for consumers and $j=p$ for producers. Let $\varphi / \phi$ denote the price of bonds in terms of money in the financial market. Then, an active agent's budget constraint satisfies

$$
\phi m_{j}+\varphi b_{j}=\phi \hat{m}_{j}+\varphi \hat{b}_{j} .
$$

The left-hand side of (37) is the sum of the real values of money and bonds with which the agent enters the financial market, and the right-hand side is the real value of the portfolio with which the agent leaves the financial market. It is obvious that a real price of bonds of $\varphi>1-g$ does not support trades in the financial market, since it is suboptimal for producers to trade. If $\varphi<1-g$, a producer's money constraint must be binding in the financial market, since it is suboptimal for 
producers not to trade all their money for bonds. However, this contradicts the characterization of the type-A and the type-B equilibria. Therefore, we only consider the case in which $\varphi=1-g$. Trading is further constrained by two short-selling constraints: Active agents cannot sell more bonds, and they cannot spend more money than the amount they carry from the previous period. That is

$$
\hat{m}_{j} \geq 0, \hat{b}_{j} \geq 0
$$

Let $V_{1}^{j}\left(m_{j}, b_{j}\right)$ denote the value function of a consumer and a producer by holding $\left(m_{j}, b_{j}\right)$ before entering the financial market. For $\varphi=1-g$, producers are indifferent between trading in the financial market or not; i.e.,

$$
V_{1}^{p}\left(m_{p}, b_{p}\right)=V_{2}^{p}\left(m_{p}, b_{p}\right)=V_{2}^{p}\left(\hat{m}_{p}, \phi m_{p} / \varphi+b_{p}-\phi \hat{m}_{p} / \varphi\right)
$$

for any $\hat{m}_{p} \geq 0, \hat{b}_{p}=\phi m_{p} / \varphi+b_{p}-\phi \hat{m}_{p} / \varphi \geq 0$. Therefore, the decision problem is up to consumers; i.e.,

$$
V_{1}^{c}\left(m_{c}, b_{c}\right)=(1-\delta) V_{2}^{c}\left(m_{c}, b_{c}\right)+\delta \max _{\hat{m}_{c}} V_{2}^{c}\left(\hat{m}_{c}, \phi m_{c} / \varphi+b_{c}-\phi \hat{m}_{c} / \varphi\right)
$$

such that $\hat{m}_{c} \geq 0, \hat{b}_{c}=\phi m_{c} / \varphi+b_{c}-\phi \hat{m}_{c} / \varphi \geq 0$. Note that

$$
\frac{d V_{2}^{c}\left(\hat{m}_{c}, \phi m_{c} / \varphi+b_{c}-\phi \hat{m}_{c} / \varphi\right)}{d \hat{m}_{c}}= \begin{cases}0 & \text { if } \phi \hat{m}_{c}>q^{*} \\ \phi u^{\prime}\left(\phi \hat{m}_{c}\right)-\phi>0 & \text {,if } \phi \hat{m}_{c}<q^{*}\end{cases}
$$

Thus, the optimal $\hat{m}_{c}$ is

$$
\hat{m}_{c} \begin{cases}\in\left[\frac{q^{*}}{\phi}, m_{c}+\frac{\varphi}{\phi} b_{c}\right] & , \text { if } u^{\prime}\left(\phi m_{c}+\varphi b_{c}\right)-1<0 \\ =m_{c}+\frac{\varphi}{\phi} b_{c} & , \text { if } u^{\prime}\left(\phi m_{c}+\varphi b_{c}\right)-1 \geq 0 .\end{cases}
$$

Therefore, we can formulate the value function of consumers as

$$
V_{1}^{c}\left(m_{c}, b_{c}\right)= \begin{cases}(1-\delta) V_{2}^{c}\left(m_{c}, b_{c}\right)+\delta V_{2}^{c}\left(\frac{q^{*}}{\phi}, \frac{\phi m_{c}}{\varphi}+b_{c}-\frac{q^{*}}{\varphi}\right) & , \text { if } u^{\prime}\left(\phi m_{c}+\varphi b_{c}\right)<1 \\ (1-\delta) V_{2}^{c}\left(m_{c}, b_{c}\right)+\delta V_{2}^{c}\left(m_{c}+\frac{\varphi}{\phi} b_{c}, 0\right) & , \text { if } u^{\prime}\left(\phi m_{c}+\varphi b_{c}\right) \geq 1 .\end{cases}
$$

Type-A Equilibrium. A type-A equilibrium is characterized by $\varphi=1-g$ and $1>u^{\prime}(\phi m+\varphi b)$. It is a list $\left\{\hat{q}, q, q_{p}, \rho, \varphi, \mathfrak{b}, \ell\right\}$ satisfying (4), (11), (12), and

$$
\begin{aligned}
\varphi & =1-g, \\
1 & =u^{\prime}(\hat{q}), \\
\frac{\gamma}{\beta} & =(1-n) \delta\left[u^{\prime}(\hat{q})-u^{\prime}(q)\right]+(1-n) u^{\prime}(q)+n, \\
\rho & =\beta(1-g) .
\end{aligned}
$$


Because the cash constraint of active producers is non-binding, it follows that $\varphi=1-g$ has to hold. Equation (42) is immediate by (40). Equation (43) and (44) are derived from (39) and (40):

$$
\begin{aligned}
\frac{\partial V_{1}}{\partial m} & =(1-n) \frac{\partial V_{1}^{c}}{\partial m_{c}}+n \frac{\partial V_{1}^{p}}{\partial m_{p}} \\
& =(1-n)\left[(1-\delta) \phi u^{\prime}(q)+\delta \phi\right]+n \phi \\
& =\phi\left\{(1-n) \delta\left[u^{\prime}(\hat{q})-u^{\prime}(q)\right]+(1-n) u^{\prime}(q)+n\right\},
\end{aligned}
$$

and

$$
\begin{aligned}
\frac{\partial V_{1}}{\partial b} & =(1-n) \frac{\partial V_{1}^{c}}{\partial b_{c}}+n \frac{\partial V_{1}^{p}}{\partial b_{p}} \\
& =(1-n)(1-g)[(1-\delta)+\delta]+n(1-g) \\
& =(1-g) .
\end{aligned}
$$

Type-B Equilibrium. A type-B equilibrium is characterized by $\varphi=1-g$ and $1 \leq u^{\prime}(\phi m+\varphi b)$. It is a list $\left\{\hat{q}, q, q_{p}, \rho, \varphi, \mathfrak{b}, \ell\right\}$ satisfying (4), (11), (12), and

$$
\begin{aligned}
\varphi & =1-g, \\
(1-g) \ell \vec{b} & =\hat{q}-q, \\
\frac{\gamma}{\beta} & =(1-n)\left[\delta u^{\prime}(\hat{q})+(1-\delta) u^{\prime}(q)\right]+n, \\
\rho & =\beta(1-g)\left\{1+(1-n) \delta\left[u^{\prime}(\hat{q})-1\right]\right\} .
\end{aligned}
$$

Because the cash constraint of active producers is non-binding, it follows that $\varphi=1-g$ has to hold. Equation (46) comes from the fact that active consumers sell all their bonds to active producers for the money they will receive, so that

$$
\phi \hat{m}=\phi m+\varphi b \quad \Rightarrow \quad \varphi \ell \vec{b}=\varphi b=\phi \hat{m}-\phi m=\hat{q}-q .
$$

Equation (47) and (48) are derived from (39) and (40):

$$
\begin{aligned}
\frac{\partial V_{1}}{\partial m} & =(1-n) \frac{\partial V_{1}^{c}}{\partial m_{c}}+n \frac{\partial V_{1}^{p}}{\partial m_{p}} \\
& =(1-n)\left[(1-\delta) \phi u^{\prime}(q)+\delta \phi u^{\prime}(\hat{q})\right]+n \phi \\
& =\phi\left[(1-n) \delta u^{\prime}(\hat{q})+(1-n)(1-\delta) u^{\prime}(q)+n\right]
\end{aligned}
$$


and

$$
\begin{aligned}
\frac{\partial V_{1}}{\partial b} & =(1-n) \frac{\partial V_{1}^{c}}{\partial b_{c}}+n \frac{\partial V_{1}^{p}}{\partial b_{p}} \\
& =(1-n)\left[(1-\delta)(1-g)+\delta(1-g) u^{\prime}(\hat{q})\right]+n(1-g) \\
& =(1-g)\left\{1+(1-n) \delta\left[u^{\prime}(\hat{q})-1\right]\right\} .
\end{aligned}
$$

Proof of Proposition 7. From (17), we obtain

$$
\begin{aligned}
& \delta u^{\prime \prime}(\hat{q}) \frac{\partial \hat{q}}{\partial \eta} \frac{\eta+(1-\eta) u^{\prime}(q)}{\eta+(1-\eta) u^{\prime}(\hat{q})}+\delta u^{\prime}(\hat{q}) \frac{1-u^{\prime}(q)}{\eta+(1-\eta) u^{\prime}(\hat{q})}+\delta u^{\prime}(\hat{q}) \frac{(1-\eta) u^{\prime \prime}(q)}{\eta+(1-\eta) u^{\prime}(\hat{q})} \frac{\partial q}{\partial \eta} \\
& -\delta u^{\prime}(\hat{q}) \frac{\eta+(1-\eta) u^{\prime}(q)}{\left[\eta+(1-\eta) u^{\prime}(\hat{q})\right]^{2}}\left[1-u^{\prime}(\hat{q})+(1-\eta) u^{\prime \prime}(\hat{q}) \frac{\partial \hat{q}}{\partial \eta}\right]+(1-\delta) u^{\prime \prime}(q) \frac{\partial q}{\partial \eta}=0
\end{aligned}
$$

Hence if $\delta \eta \neq 1$, then

$$
\begin{gathered}
\frac{\partial q}{\partial \eta}=-\frac{\delta u^{\prime}(\hat{q}) \frac{1-u^{\prime}(q)}{\eta+(1-\eta) u^{\prime}(\hat{q})}-\delta u^{\prime}(\hat{q}) \frac{\eta+(1-\eta) u^{\prime}(q)}{\left[\eta+(1-\eta) u^{\prime}(\hat{q})\right]^{2}}\left[1-u^{\prime}(\hat{q})\right]}{\delta u^{\prime}(\hat{q}) \frac{(1-\eta) u^{\prime \prime}(q)}{\eta+(1-\eta) u^{\prime}(\hat{q})}+(1-\delta) u^{\prime \prime}(q)} \\
-\frac{\delta u^{\prime \prime}(\hat{q}) \frac{\eta+(1-\eta) u^{\prime}(q)}{\eta+(1-\eta) u^{\prime}(\hat{q})}-\delta u^{\prime}(\hat{q}) \frac{\eta+(1-\eta) u^{\prime}(q)}{\left[\eta+(1-\eta) u^{\prime}(\hat{q})\right]^{2}}(1-\eta) u^{\prime \prime}(\hat{q})}{\delta u^{\prime}(\hat{q}) \frac{(1-\eta) u^{\prime \prime}(q)}{\eta+(1-\eta) u^{\prime}(\hat{q})}+(1-\delta) u^{\prime \prime}(q)} \frac{\partial \hat{q}}{\partial \eta}
\end{gathered}
$$

If $\delta \eta=1$, then

$$
\frac{\partial \hat{q}}{\partial \eta}=\frac{u^{\prime}(\hat{q})\left[u^{\prime}(q)-u^{\prime}(\hat{q})\right]}{u^{\prime \prime}(\hat{q})} .
$$

The effect on the demand of bonds is

$$
\begin{aligned}
\frac{\partial\{(1-\eta)[u(\hat{q})-u(q)]+\eta(\hat{q}-q)\}}{\partial \eta}= & -[u(\hat{q})-u(q)]+\hat{q}-q \\
& +\left[\eta+(1-\eta) u^{\prime}(\hat{q})\right] \frac{\partial \hat{q}}{\partial \eta}-\left[\eta+(1-\eta) u^{\prime}(q)\right] \frac{\partial q}{\partial \eta}
\end{aligned}
$$

From (18), we obtain

$$
\frac{\partial \rho}{\partial \eta}=\beta(1-g)(1-n) \delta\left\{\begin{array}{c}
\frac{u^{\prime}(\hat{q})-1}{\eta+(1-\eta) u^{\prime}(\hat{q})}\left[1+\frac{\eta\left[u^{\prime}(\hat{q})-1\right]}{\eta+(1-\eta) u^{\prime}(\hat{q})}\right] \\
+\eta \frac{u^{\prime \prime}(\hat{q})}{\eta+(1-\eta) u^{\prime}(\hat{q})}\left[1-\frac{\left[u^{\prime}(\hat{q})-1\right](1-\eta)}{\eta+(1-\eta) u^{\prime}(\hat{q})}\right] \frac{\partial \hat{q}}{\partial \eta}
\end{array}\right\} .
$$


From Lemma 4, we know that the effect on the supply of bonds is

$$
\frac{\partial(\ell \mathfrak{b})}{\partial \eta}=\frac{\partial(\ell \mathfrak{b})}{\partial \rho} \frac{\partial \rho}{\partial \eta}=\frac{f^{\prime}(\rho \mathfrak{b}) \mathfrak{b}}{\alpha(\rho \mathbb{b})}[1-\epsilon(\rho \mathbb{b})][1-\alpha(\rho \mathfrak{b})] \times \frac{\partial \rho}{\partial \eta},
$$

where $\partial \rho / \partial \eta$ is given by (51). By market clearing, we obtain

$$
\begin{aligned}
& -[u(\hat{q})-u(q)]+\hat{q}-q+\left[\eta+(1-\eta) u^{\prime}(\hat{q})\right] \frac{\partial \hat{q}}{\partial \eta}-\left[\eta+(1-\eta) u^{\prime}(q)\right] \frac{\partial q}{\partial \eta} \\
& =\frac{f^{\prime}(\rho \mathfrak{b}) \mathfrak{b}}{\alpha(\rho \mathfrak{b})}[1-\epsilon(\rho \mathfrak{b})][1-\alpha(\rho \mathfrak{b})] \\
& \times \beta(1-g)^{2}(1-n) \delta\left\{\begin{array}{c}
\frac{u^{\prime}(\hat{q})-1}{\eta+(1-\eta) u^{\prime}(\hat{q})}\left[1+\frac{\eta\left[u^{\prime}(\hat{q})-1\right]}{\eta+(1-\eta) u^{\prime}(\hat{q})}\right] \\
+\eta \frac{u^{\prime \prime}(\hat{q})}{\eta+(1-\eta) u^{\prime}(\hat{q})}\left[1-\frac{\left[u^{\prime}(\hat{q})-1\right](1-\eta)}{\eta+(1-\eta) u^{\prime}(\hat{q})}\right] \frac{\partial \hat{q}}{\partial \eta}
\end{array}\right\} .
\end{aligned}
$$

With (49), (50), and (52), we can explicitly derive $\partial \hat{q} / \partial \eta$, and $\partial q / \partial \eta$. For instance, if $\delta \eta=1$, then we have

$$
\begin{aligned}
\frac{\partial \rho}{\partial \eta}= & \beta(1-g)(1-n) u^{\prime}(\hat{q})\left[u^{\prime}(q)-1\right]>0 \\
\frac{\partial(\ell \mathbb{b})}{\partial \eta}= & \frac{f^{\prime}(\rho \mathfrak{b}) \mathfrak{b}}{\alpha(\rho \mathfrak{b})}[1-\epsilon(\rho \mathfrak{b})][1-\alpha(\rho \mathfrak{b})] \beta(1-g)(1-n) u^{\prime}(\hat{q})\left[u^{\prime}(q)-1\right]>0 \\
\frac{\partial q}{\partial \eta}= & -[u(\hat{q})-u(q)]+\hat{q}-q+\frac{u^{\prime}(\hat{q})\left[u^{\prime}(q)-u^{\prime}(\hat{q})\right]}{u^{\prime \prime}(\hat{q})} \\
& -\frac{f^{\prime}(\rho \mathfrak{b}) \mathbb{b}}{\alpha(\rho \mathfrak{b})}[1-\epsilon(\rho \mathbb{b})][1-\alpha(\rho \mathfrak{b})] \beta(1-g)^{2}(1-n) u^{\prime}(\hat{q})\left[u^{\prime}(q)-1\right]<0 .
\end{aligned}
$$

By the continuity, if the values of $\delta$ and $\eta$ are large enough, then

$$
\frac{\partial \rho}{\partial \eta}>0, \quad \frac{\partial(\ell b)}{\partial \eta}>0, \quad \frac{\partial \hat{q}}{\partial \eta}<0, \quad \frac{\partial q}{\partial \eta}<0 .
$$

Next, we derive the effects of a change in $\eta$ on welfare. From (4), we have

$$
n \frac{\partial q_{p}}{\partial \eta}=(1-n)\left[\delta \frac{\partial \hat{q}}{\partial \eta}+(1-\delta) \frac{\partial q}{\partial \eta}\right] .
$$

Hence, together with (20), we obtain

$$
\begin{aligned}
(1-\beta) \frac{\partial \mathcal{W}}{\partial \eta} & =(1-n)\left[\delta u^{\prime}(\hat{q}) \frac{\partial \hat{q}}{\partial \eta}+(1-\delta) u^{\prime}(q) \frac{\partial q}{\partial \eta}\right]-n \frac{\partial q_{p}}{\partial \eta} \\
& =(1-n)\left[\delta\left[u^{\prime}(\hat{q})-1\right] \frac{\partial \hat{q}}{\partial \eta}+(1-\delta)\left[u^{\prime}(q)-1\right] \frac{\partial q}{\partial \eta}\right]<0 .
\end{aligned}
$$


Therefore, if $\delta \eta=1$, then $\partial \mathcal{W} / \partial \eta<0$.

Proof of Corollary 8. Consider $\tilde{\eta}<\eta$ such that $\hat{q}=q^{*}$ in the type-B equilibrium; i.e., $\gamma, \delta$, and $\tilde{\eta}$ would support both the type-A and the type-B equilibrium. The equation (49) and the equation (52) imply that

$$
\begin{aligned}
& \delta \tilde{\eta} u^{\prime \prime}(\hat{q})\left[\tilde{\eta}+(1-\tilde{\eta}) u^{\prime}(q)\right] \frac{\partial \hat{q}}{\partial \eta}+(1-\delta \tilde{\eta}) u^{\prime \prime}(q) \frac{\partial q}{\partial \eta}=-\delta\left[1-u^{\prime}(q)\right]>0, \\
& \left\{1-\frac{f^{\prime}(\rho \mathbb{b}) \mathbb{b}}{\alpha(\rho \mathbb{b})}[1-\epsilon(\rho \mathbb{b})][1-\alpha(\rho \mathbb{b})] \beta(1-g)^{2}(1-n) \delta \tilde{\eta} u^{\prime \prime}(\hat{q})\right\} \frac{\partial \hat{q}}{\partial \eta}-\left[\tilde{\eta}+(1-\tilde{\eta}) u^{\prime}(q)\right] \frac{\partial q}{\partial \eta} \\
& =[1-u(q)]-\hat{q}+q \geq 0 .
\end{aligned}
$$

From the above two equations, at $\tilde{\eta}$, we obtain

$$
\frac{\partial q}{\partial \eta}<0
$$

and

$$
(1-\beta) \frac{\partial \mathcal{W}}{\partial \eta}=(1-n)(1-\delta)\left[u^{\prime}(q)-1\right] \frac{\partial q}{\partial \eta}<0 .
$$

By our assumption, $\tilde{\eta}$ is close to $\eta$, hence $\partial \mathcal{W} / \partial \eta<0$ for $\eta>\tilde{\eta}$ by continuity.

Proof of Proposition 9. From (4) and (14), we obtain

$$
\begin{aligned}
n \frac{\partial q_{p}}{\partial \eta} & =(1-n)(1-\delta) \frac{\partial q}{\partial \eta} \\
\frac{\partial q}{\partial \eta} & =\frac{\delta\left[u^{\prime}(q)-1\right]}{(1-\delta \eta) u^{\prime \prime}(q)} .
\end{aligned}
$$

Using these two expressions in (20), we have

$$
\begin{aligned}
(1-\beta) \frac{\partial \mathcal{W}}{\partial \eta} & =(1-n)(1-\delta) u^{\prime}(q) \frac{\partial q}{\partial \eta}-n \frac{\partial q_{p}}{\partial \eta} \\
& =\frac{(1-n)(1-\delta) \delta\left[u^{\prime}(q)-1\right]^{2}}{(1-\delta \eta) u^{\prime \prime}(q)}<0 .
\end{aligned}
$$

Proof of Proposition 10. From (17), we obtain

$$
\begin{aligned}
& u^{\prime}(\hat{q}) \frac{\eta+(1-\eta) u^{\prime}(q)}{\eta+(1-\eta) u^{\prime}(\hat{q})}-u^{\prime}(q)+\delta \frac{\eta+(1-\eta) u^{\prime}(q)}{\eta+(1-\eta) u^{\prime}(\hat{q})} u^{\prime \prime}(\hat{q}) \frac{\partial \hat{q}}{\partial \delta} \\
& +\delta u^{\prime}(\hat{q}) \frac{(1-\eta) u^{\prime \prime}(q)}{\left[\eta+(1-\eta) u^{\prime}(\hat{q})\right]} \frac{\partial q}{\partial \delta}-\delta u^{\prime}(\hat{q}) \frac{\left[\eta+(1-\eta) u^{\prime}(q)\right](1-\eta) u^{\prime \prime}(\hat{q})}{\left[\eta+(1-\eta) u^{\prime}(\hat{q})\right]^{2}} \frac{\partial \hat{q}}{\partial \delta}+(1-\delta) u^{\prime \prime}(q) \frac{\partial q}{\partial \delta}=0 .
\end{aligned}
$$


Hence, if $\delta \eta \neq 1$, then we obtain

$$
\frac{\partial q}{\partial \delta}=\frac{u^{\prime}(q)-u^{\prime}(\hat{q}) \frac{\eta+(1-\eta) u^{\prime}(q)}{\eta+(1-\eta) u^{\prime}(\hat{q})}-\delta \frac{\eta+(1-\eta) u^{\prime}(q)}{\eta+(1-\eta) u^{\prime}(\hat{q})} u^{\prime \prime}(\hat{q})\left[1-\frac{(1-\eta) u^{\prime}(\hat{q})}{\eta+(1-\eta) u^{\prime}(\hat{q})}\right] \frac{\partial \hat{q}}{\partial \delta}}{\left\{\delta u^{\prime}(\hat{q}) \frac{(1-\eta)}{\left[\eta+(1-\eta) u^{\prime}(\hat{q})\right]}+(1-\delta)\right\} u^{\prime \prime}(q)}
$$

If $\delta \eta=1$, then we obtain

$$
\frac{\partial \hat{q}}{\partial \delta}=\frac{u^{\prime}(q)-u^{\prime}(\hat{q})}{u^{\prime \prime}(\hat{q})}<0 .
$$

The effect on the demand of bonds is

$$
\frac{\partial\{(1-\eta)[u(\hat{q})-u(q)]+\eta(\hat{q}-q)\}}{\partial \delta}=\left[\eta+(1-\eta) u^{\prime}(\hat{q})\right] \frac{\partial \hat{q}}{\partial \delta}-\left[\eta+(1-\eta) u^{\prime}(q)\right] \frac{\partial q}{\partial \delta} .
$$

From (18), we have

$$
\frac{\partial \rho}{\partial \delta}=\beta(1-g)(1-n) \eta\left\{\frac{u^{\prime}(\hat{q})-1}{\eta+(1-\eta) u^{\prime}(\hat{q})}+\delta \frac{u^{\prime \prime}(\hat{q})}{\eta+(1-\eta) u^{\prime}(\hat{q})}\left[1-\frac{\left[u^{\prime}(\hat{q})-1\right](1-\eta)}{\eta+(1-\eta) u^{\prime}(\hat{q})}\right] \frac{\partial \hat{q}}{\partial \delta}\right\} .
$$

From Lemma 4, we know that the effect on the supply of bonds is

$$
\frac{\partial(\ell \mathfrak{b})}{\partial \delta}=\frac{\partial(\ell \mathfrak{b})}{\partial \rho} \frac{\partial \rho}{\partial \delta}=\frac{f^{\prime}(\rho \mathfrak{b}) \mathfrak{b}}{\alpha(\rho \mathfrak{b})}[1-\epsilon(\rho \mathfrak{b})][1-\alpha(\rho \mathfrak{b})] \times \frac{\partial \rho}{\partial \delta},
$$

where $\partial \rho / \partial \delta$ is given by (55). By market clearing, we have

$$
\begin{aligned}
& {\left[\eta+(1-\eta) u^{\prime}(\hat{q})\right] \frac{\partial \hat{q}}{\partial \delta}-\left[\eta+(1-\eta) u^{\prime}(q)\right] \frac{\partial q}{\partial \delta} } \\
= & \frac{f^{\prime}(\rho \mathfrak{b}) \mathfrak{b}}{\alpha(\rho \mathfrak{b})}[1-\epsilon(\rho \mathfrak{b})][1-\alpha(\rho \mathfrak{b})] \\
& \times \beta(1-n)(1-g)^{2} \eta\left\{\frac{u^{\prime}(\hat{q})-1}{\eta+(1-\eta) u^{\prime}(\hat{q})}+\delta \frac{u^{\prime \prime}(\hat{q})}{\eta+(1-\eta) u^{\prime}(\hat{q})}\left[1-\frac{\left[u^{\prime}(\hat{q})-1\right](1-\eta)}{\eta+(1-\eta) u^{\prime}(\hat{q})}\right] \frac{\partial \hat{q}}{\partial \delta}\right\} .
\end{aligned}
$$

With (53), (54), and (56), we can explicitly derive $\partial \hat{q} / \partial \delta$, and $\partial q / \partial \delta$. For instance, if $\delta \eta=1$, then we have

$$
\begin{aligned}
\frac{\partial \rho}{\partial \delta} & =\beta(1-g)(1-n)\left[u^{\prime}(q)-1\right]>0 \\
\frac{\partial(\ell \mathbb{b})}{\partial \delta} & =\frac{f^{\prime}(\rho \mathfrak{b}) \mathfrak{b}}{\alpha(\rho \mathfrak{b})}[1-\epsilon(\rho \mathfrak{b})][1-\alpha(\rho \mathfrak{b})] \beta(1-g)(1-n)\left[u^{\prime}(q)-1\right]>0 \\
\frac{\partial q}{\partial \delta} & =\frac{u^{\prime}(q)-u^{\prime}(\hat{q})}{u^{\prime \prime}(\hat{q})}-\frac{f^{\prime}(\rho \mathfrak{b}) \mathfrak{b}}{\alpha(\rho \mathfrak{b})}[1-\epsilon(\rho \mathfrak{b})][1-\alpha(\rho \mathbb{b})] \beta(1-g)^{2}(1-n)\left[u^{\prime}(q)-1\right]<0 .
\end{aligned}
$$


By the continuity, if the values of $\delta$ and $\eta$ are large enough, then

$$
\frac{\partial \rho}{\partial \delta}>0, \quad \frac{\partial(\ell \mathfrak{b})}{\partial \delta}>0, \quad \frac{\partial \hat{q}}{\partial \delta}<0, \quad \frac{\partial q}{\partial \delta}<0 .
$$

Next, we derive the effects of a change in $\delta$ on welfare. From (4), we have

$$
n \frac{\partial q_{p}}{\partial \delta}=(1-n)\left[\delta \frac{\partial \hat{q}}{\partial \delta}+(1-\delta) \frac{\partial q}{\partial \delta}+\hat{q}-q\right] .
$$

Hence, together with (20), we obtain

$$
\begin{aligned}
(1-\beta) \frac{\partial \mathcal{W}}{\partial \delta} & =(1-n)\left[\delta u^{\prime}(\hat{q}) \frac{\partial \hat{q}}{\partial \delta}+(1-\delta) u^{\prime}(q) \frac{\partial q}{\partial \delta}+u(\hat{q})-u(q)\right]-n \frac{\partial q_{p}}{\partial \delta} \\
& =(1-n)\left\{\delta\left[u^{\prime}(\hat{q})-1\right] \frac{\partial \hat{q}}{\partial \delta}+(1-\delta)\left[u^{\prime}(q)-1\right] \frac{\partial q}{\partial \delta}+u(\hat{q})-u(q)-(\hat{q}-q)\right\} .
\end{aligned}
$$

Note that if $\delta \eta=1$, then

$$
\begin{aligned}
\frac{1-\beta}{1-n} \frac{\partial \mathcal{W}}{\partial \delta} & =\frac{\left[u^{\prime}(q)-u^{\prime}(\hat{q})\right]\left[u^{\prime}(\hat{q})-1\right]}{u^{\prime \prime}(\hat{q})}+u(\hat{q})-u(q)-(\hat{q}-q) \\
& =(\hat{q}-q)\left[\frac{u(\hat{q})-u(q)}{\hat{q}-q}-\frac{u^{\prime}(\hat{q})-u^{\prime}(q)}{\hat{q}-q} \frac{u^{\prime}(\hat{q})-1}{u^{\prime \prime}(\hat{q})}-1\right] .
\end{aligned}
$$

Furthermore, note that

$$
\frac{u\left(q^{*}\right)-u(q)}{q^{*}-q}-\frac{u^{\prime}\left(q^{*}\right)-u^{\prime}(q)}{q^{*}-q} \frac{u^{\prime}\left(q^{*}\right)-1}{u^{\prime \prime}\left(q^{*}\right)}-1=\frac{u\left(q^{*}\right)-u(q)}{q^{*}-q}-1>0,
$$

so by continuity, if $\gamma$ is not large (but $\delta$ and $\eta$ are large), then we have

$$
\frac{u(\hat{q})-u(q)}{\hat{q}-q}-\frac{u^{\prime}(\hat{q})-u^{\prime}(q)}{\hat{q}-q} \frac{u^{\prime}(\hat{q})-1}{u^{\prime \prime}(\hat{q})}-1>0 ;
$$

i.e., welfare is improving as $\delta$ increases $(\partial \mathcal{W} / \partial \delta>0)$, even if all agents consume less with a higher value of $\delta$.

Proof of Corollary 11. From the proof of Proposition 10, if $\delta \eta=1$, then

$$
\frac{1-\beta}{1-n} \frac{\partial \mathcal{W}}{\partial \delta}=\frac{\left[u^{\prime}(q)-u^{\prime}(\hat{q})\right]\left[u^{\prime}(\hat{q})-1\right]}{u^{\prime \prime}(\hat{q})}+u(\hat{q})-u(q)-(\hat{q}-q) .
$$

Now let $u(q)=\ln q$. Then we have

$$
\frac{1-\beta}{1-n} \frac{\partial \mathcal{W}}{\partial \delta}=-\left(\frac{\hat{q}}{q}-1\right)(1-\hat{q})+\ln \frac{\hat{q}}{q}-(\hat{q}-q)
$$


As $\gamma$ goes to infinitely large, we have

$$
\lim _{\gamma \rightarrow \infty} \frac{1-\beta}{1-n} \frac{\partial \mathcal{W}}{\partial \delta}=-\left(q_{r}-1\right)+\ln q_{r}
$$

for some $q_{r}:=\lim _{\gamma \rightarrow \infty} \hat{q} / q$. The proof is completed by noticing that

$$
-\left(q_{r}-1\right)+\ln q_{r}<0 .
$$

By continuity, it also holds for large $\delta$ and $\eta$.

Proof of Proposition 12. From (4) and (14), we obtain

$$
\begin{aligned}
n \frac{\partial q_{p}}{\partial \delta} & =(1-n)(1-\delta) \frac{\partial q}{\partial \delta}+(1-n)(\hat{q}-q), \\
\frac{\partial q}{\partial \delta} & =\frac{\eta\left[u^{\prime}(q)-1\right]}{(1-\delta \eta) u^{\prime \prime}(q)} .
\end{aligned}
$$

Using these two expressions in (20), we obtain

$$
\begin{aligned}
(1-\beta) \frac{\partial \mathcal{W}}{\partial \delta} & =(1-n)(1-\delta) u^{\prime}(q) \frac{\partial q}{\partial \delta}-n \frac{\partial q_{p}}{\partial \delta}+(1-n)[u(\hat{q})-u(q)] \\
& =\frac{(1-n)(1-\delta) \eta\left[u^{\prime}(q)-1\right]^{2}}{(1-\delta \eta) u^{\prime \prime}(q)}+(1-n)[u(\hat{q})-u(q)-(\hat{q}-q)] .
\end{aligned}
$$

If $\eta=0$, then

$$
(1-\beta) \frac{\partial \mathcal{W}}{\partial \delta}=(1-n)[u(\hat{q})-u(q)-(\hat{q}-q)]>0,
$$

because

$$
u(\hat{q})-u(q)-(\hat{q}-q)=\frac{1}{\hat{q}-q}\left[\frac{u(\hat{q})-u(q)}{\hat{q}-q}-1\right]>\frac{1}{\hat{q}-q}\left[u^{\prime}(\hat{q})-1\right]=0 .
$$

Thus, if $\eta$ is close to zero, then it is optimal to increase $\delta$. Now consider the case with $\eta=1$, where we have

$$
\frac{1-\beta}{1-n} \frac{\partial \mathcal{W}}{\partial \delta}=\frac{\left[u^{\prime}(q)-1\right]^{2}}{u^{\prime \prime}(q)}+[u(\hat{q})-u(q)-(\hat{q}-q)] .
$$

Note that

$$
\frac{\left[u^{\prime}(q)-1\right]^{2}}{u^{\prime \prime}(q)}+[u(\hat{q})-u(q)-(\hat{q}-q)]=0
$$


at $q=\hat{q}$, and

$$
\begin{aligned}
\frac{\partial}{\partial q} & \left\{\frac{\left[u^{\prime}(q)-1\right]^{2}}{u^{\prime \prime}(q)}+[u(\hat{q})-u(q)-(\hat{q}-q)]\right\} \\
& =\frac{2\left[u^{\prime}(q)-1\right]\left[u^{\prime \prime}(q)\right]^{2}-\left[u^{\prime}(q)-1\right]^{2} u^{\prime \prime \prime}(q)}{\left[u^{\prime \prime}(q)\right]^{2}}-\left[u^{\prime}(q)-1\right] \\
& =\left[u^{\prime}(q)-1\right]\left\{1-\frac{\left[u^{\prime}(q)-1\right] u^{\prime \prime \prime}(q)}{\left[u^{\prime \prime}(q)\right]^{2}}\right\}>0
\end{aligned}
$$

for $q$ close to $\hat{q}$. Therefore, we have

$$
\frac{\partial \mathcal{W}}{\partial \delta}<0
$$

for $\gamma$ close to $\beta$.

Proof of Lemma 13. First, we consider the case with $\delta=0$, which supports the type-A equilibrium, $\hat{q}_{A}, q_{A}, q_{p, A}$. Using the clearing condition (4), we have $(1-n) q_{A}=n q_{p, A}$, so that welfare with $\delta=0$ is given by

$$
\begin{aligned}
\left.(1-\beta) \mathcal{W}\right|_{\delta=0} & =U\left(x^{*}\right)-x^{*}+(1-n) u\left(q_{A}\right)-n q_{p, A} \\
& =U\left(x^{*}\right)-x^{*}+(1-n)\left[u\left(q_{A}\right)-q_{A}\right],
\end{aligned}
$$

where $q_{A}$ satisfies $\gamma / \beta=(1-n) u^{\prime}\left(q_{A}\right)+n$ by (14). Now, consider the case with $\delta=1$, which supports the type-B equilibrium, $\hat{q}_{B}, q_{B}, q_{p, B}$. By (4), we have $(1-n) \hat{q}_{B}=n q_{p, B}$, so that welfare with $\delta=1$ is given by

$$
\begin{aligned}
\left.(1-\beta) \mathcal{W}\right|_{\delta=1} & =U\left(x^{*}\right)-x^{*}+(1-n) u\left(\hat{q}_{B}\right)-n q_{p, B} \\
& =U\left(x^{*}\right)-x^{*}+(1-n)\left[u\left(\hat{q}_{B}\right)-\hat{q}_{B}\right],
\end{aligned}
$$

where $\hat{q}_{B}$ satisfies $\gamma / \beta=(1-n) u^{\prime}\left(\hat{q}_{B}\right)\left[\eta+(1-\eta) u^{\prime}\left(q_{B}\right)\right] /\left[\eta+(1-\eta) u^{\prime}\left(\hat{q}_{B}\right)\right]+n$ by (17). Note that $\left[\eta+(1-\eta) u^{\prime}\left(q_{B}\right)\right] /\left[\eta+(1-\eta) u^{\prime}\left(\hat{q}_{B}\right)\right]>1$ for $\eta<1$ and $\left[\eta+(1-\eta) u^{\prime}\left(q_{B}\right)\right] /\left[\eta+(1-\eta) u^{\prime}\left(\hat{q}_{B}\right)\right]=$ 1 for $\eta=1$. Therefore, $\hat{q}_{B}>q_{A}$ for $\eta<1$, and $\hat{q}_{B}=q_{A}$ for $\eta=1$. The proof is completed by noticing that $u(x)-x$ is concave and maximized at $q^{*} \geq \hat{q}_{B}$.

Proof of Lemma 14. Note that, given $\gamma, \beta, \eta, n, C$, and $g$, if $x$ satisfies

$$
\frac{\gamma}{\beta}=(1-n) u^{\prime}(x)+n,
$$

then $\hat{q}_{A}:=q^{*}, q_{A}:=x, q_{A p}=(1-n) x / n$ support the type-A equilibrium when $\delta=\tilde{\delta}:=0$, which is equivalent to having no financial market at all. If over-the-counter trading is superior 
to having no financial market at all, then we must have

$$
\begin{aligned}
\left.(1-\beta) \mathcal{W}\right|_{\delta, \eta} & =U\left(x^{*}\right)-x^{*}+(1-n)[\delta u(\hat{q})+(1-\delta) u(q)]-n q_{p} \\
& >U\left(x^{*}\right)-x^{*}+(1-n)\left[\tilde{\delta} u\left(q^{*}\right)+(1-\tilde{\delta}) u\left(q_{A}\right)\right]-n q_{A p}=\left.(1-\beta) \mathcal{W}\right|_{\tilde{\delta}=0},
\end{aligned}
$$

which is equivalent to

$$
\frac{u(x)-x-[u(q)-q]}{u(\hat{q})-\hat{q}-[u(q)-q]}<\delta
$$

\section{References}

[1] Acharya, V., and Bisin, A., 2014, "Counterparty Risk Externality: Centralized versus OverThe-Counter Markets", Journal of Economic Theory, 149, 153-182.

[2] Andolfatto, D., 2011, "A Note on the Societal Benefits of Illiquid Bonds", Canadian Journal of Economics, 44, 133-147.

[3] Aruoba, B., Rocheteau, G., and Waller, C., 2007, "Bargaining and the Value of Money", Journal of Monetary Economics, 54, 2636-2655.

[4] Berentsen, A., Camera, G., and Waller, C., 2007, "Money, Credit and Banking", Journal of Economic Theory, 135, 171-195.

[5] Berentsen, A., Huber, S., and Marchesiani, A., 2014, "Degreasing the Wheels of Finance", International Economic Review, 55, 735-763.

[6] Berentsen, A., Huber, S., and Marchesiani, A., 2016, "The Societal Benefit of a Financial Transaction Tax", European Economic Review, 89, 303-323.

[7] Berentsen, A., Menzio, G., and Wright, R., 2011, "Inflation and Unemployment in the Long Run", American Economic Review, 101, 371-398.

[8] Berentsen, A., and Waller, C., 2011, "Outside Versus Inside Bonds: A Modigliani-Miller Type Result for Liquidity Constrained Economies", Journal of Economic Theory, 146, 18521887.

[9] Branch, W. A., Petrosky-Nadeau, N., and Rocheteau, G., 2016, "Financial Frictions, the Housing Market, and Unemployment", Journal of Economic Theory, 164, 101-135.

[10] Chun, Y., and Thomson, W., 1988, "Monotonicity Properties of Bargaining Solutions When Applied to Economics", Mathematical Social Sciences, 15, 11-27.

[11] Duffie, D., 2012, "Dark Markets: Asset Pricing and Information Transmission in Over-theCounter Markets", Princeton Lectures in Finance. 
[12] Duffie, D., Gârleanu, N., and Pederson, L. H., 2005, "Over-the-Counter Markets", Econometrica, $73,1815-1847$.

[13] Duffie, D., and Zhu, H., 2011, "Does a Central Clearing Counterparty Reduce Counterparty Risk?", Review of Asset Pricing Studies, 1, 74-95.

[14] Gallagher, D. M., 2014, "Remarks to the Georgetown University Center for Financial Markets and Policy Conference on Financial Markets Quality", SEC speeches.

[15] Geromichalos, A., and Herrenbrueck, L., 2016a, "Monetary Policy, Asset Prices, and Liquidity in Over-the-Counter Markets", Journal of Money, Credit and Banking, 28, 35-79.

[16] Geromichalos, A., and Herrenbrueck, L., 2016b, "The Strategic Determination of the Supply of Liquid Assets", working paper.

[17] Geromichalos, A., and Herrenbrueck, L., 2017, "A Tractable Model of Indirect Asset Liquidity", Journal of Economic Theory, 168, 252-260.

[18] Kalai, E., 1977, "Proportional Solutions to Bargaining Situations: Interpersonal Utility Comparisons", Econometrica, 45, 1623-1630.

[19] Kiyotaki, N., and Wright, R., 1989, "On Money As a Medium of Exchange", Journal of Political Economy, 97, 927-954.

[20] Kocherlakota, N., 1998, "Money Is Memory", Journal of Economic Theory, 81, 232-251.

[21] Kocherlakota, N., 2003, "Societal Benefits of Illiquid Bonds", Journal of Economic Theory, 108, 179-193.

[22] Lagos, R., and Rocheteau, G., 2009, "Liquidity in Asset Markets with Search Frictions", Econometrica, 77, 403-426.

[23] Lagos, R., Rocheteau, G., and Wright, R., 2015, "Liquidity: A New Monetarist Perspective", Journal of Economic Literature, forthcoming.

[24] Lagos, R., and Wright, R., 2005, "A Unified Framework for Monetary Theory and Policy Evaluation", Journal of Political Economy, 113, 463-484.

[25] Lagos, R., and Zhang, S., 2015, "Monetary Exchange in Over-the-Counter Markets: A Theory of Speculative Bubbles, the Fed Model, and Self-fulfilling Liquidity Crises", working paper (first version 2014).

[26] Leitner, Y., 2013, "Inducing Agents to Report Hidden Trades: A Theory of an Intermediary", Review of Finance, 16, 1013-1042.

[27] Mortensen, D. T., and Pissarides, C. A., 1994, "Job Creation and Job Destruction in the Theory of Unemployment", Review of Economic Studies, 61, 397-415. 
[28] Nosal, E., and Rocheteau, G., 2011, "Money, Payments, and Liquidity", MIT Press.

[29] Parlour, C., and Rajan, U., 2001, "Competition in Loan Contracts", American Economic Review, 91, 1311-1328.

[30] Rocheteau, G., and Rodriguez-Lopez, A., 2014, "Liquidity Provision, Interest Rates, and Unemployment", Journal of Monetary Economics, 65, 80-101.

[31] Rocheteau, G., and Weill, P. O., 2011, "Liquidity in Frictional Asset Markets", Journal of Money, Credit and Banking, 43, 261-282.

[32] Rocheteau, G., and Wright, R., 2005, "Money in Competitive Equilibrium, in Search Equilibrium, and in Competitive Search Equilibrium", Econometrica, 73, 175-202.

[33] Shi, S., 2006, "Viewpoint: A Microfoundation of Monetary Economics", Canadian Journal of Economics, 39, 643-688.

[34] Stephens, E., and Thompson, J., 2014, "CDS as Insurance: Leaky Lifeboats in Stormy Seas", Journal of Financial Intermediation, 23, 279-299.

[35] Thompson, J., 2010, "Counterparty Risk in Financial Contracts: Should the Insured Worry about the Insurer?", Quarterly Journal of Economics, 125, 1195-1252.

[36] Trejos, A., and Wright, R., 2014, "Search-Based Models of Money and Finance: An Integrated Approach", Journal of Economic Theory, forthcoming.

[37] Wallace, N., 2001, "Whither Monetary Economics?", International Economic Review, 42, 847-869.

[38] Williamson, S., 1987, "Financial Intermediation, Business Failures, and Real Business Cycles", Journal of Political Economy, 95, 265-302.

[39] Williamson, S., 2012, "Liquidity, Monetary Policy, and the Financial Crisis: A New Monetarist Approach", American Economic Review, 102, 2570-2605.

[40] Williamson, S., and Wright, R., 2010 "New Monetarist Economics: Models", in B. Friedman and M. Woodford (eds.) Handbook of Monetary Economics, Volume II, Amsterdam: NorthHolland. 